Revue des patrimoines

$17 \mid 2011$

Les patrimoines de l'enseignement supérieur

\title{
Le flambeau du savoir et la flamme du souvenir
}

Monuments aux morts et culte des morts dans les établissements d'enseignement supérieur 1918-1939

\section{Christian Hottin}

\section{(2) OpenEdition}

1 Journals

\section{Édition électronique}

URL : http://journals.openedition.org/insitu/984

DOI : $10.4000 /$ insitu. 984

ISSN : 1630-7305

Éditeur

Ministère de la culture

Référence électronique

Christian Hottin, «Le flambeau du savoir et la flamme du souvenir », In Situ [En ligne], 17 | 2011, mis en ligne le 05 décembre 2011, consulté le 19 avril 2019. URL : http://journals.openedition.org/insitu/984 ; DOI : 10.4000/insitu.984

Ce document a été généré automatiquement le 19 avril 2019

\section{(c) $($ i) $(9)$}

In Situ Revues des patrimoines est mis à disposition selon les termes de la licence Creative Commons Attribution - Pas d'Utilisation Commerciale - Pas de Modification 4.0 International. 


\section{Le flambeau du savoir et la flamme du souvenir}

Monuments aux morts et culte des morts dans les établissements d'enseignement supérieur 1918-1939

\section{Christian Hottin}

Fulget in gloria pretiosus sanguis eorum ${ }^{1}$

\section{Variété des monuments et diversité des formes de culte}

1 Le quatorze juillet 1919, à l'occasion du défilé qui rassembla les armées alliées sur les Champs-Élysées, la France célébra sans doute une de ses plus grandes victoires, mais elle rendit avant tout hommage à ses morts, comme en témoigne le cénotaphe érigé en haut de l'avenue et évoqué par Jules Romains dans Les Hommes de bonne volonté:

«Ils apercevaient, sous l'Arc de Triomphe, à travers les lances des dragons, le faîte du grand cénotaphe. Ils savaient par les journaux qu'il portait, en lettres énormes, l'inscription : “Aux morts pour la patrie”. L'on n'avait pas besoin de la lire soimême avec ses yeux pour en avoir l'obsession. Elle dominait l'avenue. Elle donnerait le ton au défilé. Elle en chanterait à bouche fermée le refrain principal. La fête de la Victoire allait être en premier lieu une fête des morts. Tout le monde le savait, y pensait, y consentait ${ }^{2}$.»

2 Cette commémoration, matérialisée par un monument provisoire, en attendant que la tombe du soldat inconnu prenne place sous l'Arc de Triomphe, est bien celle qui émane de l'ensemble de la communauté nationale et est destinée à tous les disparus, quelle que soit leur qualité ou le lieu de leur mort. À l'autre extrémité de cette chaîne du souvenir, voici la mémoire individuelle, la douleur ressentie à la perte d'un être cher, qui se dérobe souvent à l'historien, mais que le romancier peut saisir ; elle est l'atome, l'élément de base du culte des morts :

« Ils pensent à leurs morts ; cela se voit. Des milliers, des milliers d'entre eux précisément à tel mort, tombé à tel endroit, à tel autre, tombé à tel autre endroit. Et 
jamais évidemment il ne s'est trouvé dans une foule assemblée pour fêter la victoire autant de gens qui eussent "leurs morts" (...) Et le silence de la foule, dans toute son étendue, est soulevé ainsi de place en place par une source de sanglots plus gonflée que les autres ${ }^{3}$.»

3 Entre ces deux pôles, nous trouvons toute une suite de commémorations intermédiaires, dont divers types de monuments conservent la trace : monuments d'inspiration nationale mais élevés à l'emplacement des combats pour honorer les morts d'une bataille précise (Verdun, la Champagne, la Somme, le Chemin des Dames) ${ }^{4}$ ou monuments locaux dédiés aux combattants de tous les fronts (ceux de nos communes) . $^{5}$ Portons notre attention sur les monuments par lesquels une communauté moins évidente et moins aisément circonscrite que la commune rend un dernier hommage à ses membres tombés au front et manifeste par la même occasion son existence particulière : les monuments paroissiaux qui, à l'exception des régions animées d'un catholicisme fervent, sont distincts des monuments communaux et ont généralement l'apparence d'une plaque de marbre ${ }^{6}$ apposée sur un bas-côté de l'église, les monuments de quartier 7 , les monuments érigés par des corps professionnels particuliers ${ }^{8}$. Par le culte rendu à ses défunts, culte dont le monument n'est que l'expression la plus visible, comme la partie émergée d'un iceberg, un groupe humain marque sa spécificité, témoigne de son originalité au sein de ces ensembles naturels plus vastes que sont la Nation ou la localité ; en fait, groupée autour des disparus, la communauté se détermine et se définit.

4 C'est dans cette catégorie que nous plaçons les monuments aux morts érigés après 1918 dans les établissements d'enseignement supérieur parisiens et les diverses manifestations cultuelles qui les précédèrent ou les utilisèrent comme point d'ancrage. Communautés anciennes et dotées d'une personnalité originale, ces écoles et ces facultés le sont assurément; en 1920 plusieurs sont centenaires ou proches de l'être: Polytechnique et Normale sont les filles de la Révolution, l'École des chartes est celle de la Restauration, Centrale naquit d'une initiative privée à la fin du règne de Charles X... Mais si l'ancienneté administrative peut contribuer à fonder le sentiment d'une communauté humaine, elle n'en est pas une manifestation ; or ce sentiment s'est déjà manifesté à maintes reprises dans ces institutions : expérience personnelle des années de scolarité, commémorations et anniversaires, érections de bustes ou de monuments funéraires dédiés aux anciens maîtres, grands programmes de peintures décoratives financés par l'administration des beaux-arts et ouvrages consacrés à l'histoire de la Maison ont contribué à fonder, puis à entretenir et à enrichir le lien unissant tous ceux d'un même établissement. Ce lien est en général peu visible, simplement initié et rappelé par ces manifestations annuelles que sont les bizutages, et l'école paraît alors réduite à son enveloppe administrative ; il gagne en épaisseur et en densité dans les manifestations tristes ou gaies qui rassemblent la communauté, il prend une consistance dramatique lorsque la guerre éclate, jetant tous ses membres les plus jeunes dans le danger ${ }^{9}$, il devient enfin essentiel lorsque les vivants se rassemblent autour des morts. Dans la France de l'entre-deux-guerres, monuments aux morts et culte des morts sont les manifestations paroxystiques de l'existence des communautés issues des grandes écoles et de l'affirmation de leur spécificité au sein de la Nation.

5 En étudiant la genèse de ce culte, avant même la commande des premières œuvres, puis sa matérialisation dans le marbre, son entretien autour du monument mais aussi au-delà de ce dernier, et, pour finir, en constatant que, parfois, le monument survit au culte, nous découvrons comment ces groupes d'individus conçoivent le lien qui les unit à la communauté nationale, et comment, souvent, le sentiment de la spécificité 
communautaire relègue au second plan l'appartenance nationale pour exalter l'institution.

\section{Naissance du culte et genèse des monuments}

6 Si des cérémonies visant à honorer la mémoire des disparus de certaines écoles se tinrent durant le conflit, elles n'en restèrent pas moins exceptionnelles : toutes les institutions vivaient alors au ralenti, anciens élèves et professeurs étaient mobilisés, les concours mêmes n'étaient pas toujours organisés : il semble qu'il y ait eu quelques offices religieux, et certains donnèrent lieux à la publication de brochures, comme celle éditée par les soins de l'Association Amicale de l'Institut National Agronomique ${ }^{10}$. En fait, les premières manifestations de piété envers les morts, encore purement orales et immatérielles, semblent s'être produites à l'occasion de ces moments charnières que sont les rentrées universitaires. Étrange rentrée que celle de 1919, puisqu'elle se tint au printemps et regroupa, dans certaines écoles, des anciens dont la scolarité avait été interrompue par la mobilisation générale, les lauréats des concours de 1914 passés directement de leurs classes préparatoires au front et de tout jeunes gens que la guerre avait épargnés ${ }^{11}$. Sur les bancs des amphithéâtres de Polytechnique se massaient plusieurs dizaines de capitaines. Rue d'Ulm comme rue Descartes, le Président de la République présida la séance de rentrée et tint à chaque fois un discours ${ }^{12}$. La relation nouée entre les écoles et la Nation autour du culte de leurs morts débutait donc par un solennel hommage de son plus éminent représentant, exaltant l'ampleur et l'exemplarité du sacrifice consenti par les établissements d'enseignement supérieur: à l'École Normale Supérieure, certaines promotions, dont les plus récentes, furent décimées, perdant plus de $40 \%$ de leurs effectifs ${ }^{13}$. C'est sans aucun doute dans l'énormité des pertes qu'il faut chercher la raison de l'ampleur et de la rapidité du mouvement d'érection des monuments aux morts, comme si le choc ressenti appelait une réponse immédiate; universités et grandes écoles ne diffèrent alors guère de l'ensemble de la société française :

« Les hommes ont fait beaucoup de guerres, ont eu souvent à connaître la victoire, la défaite. Jamais, avant de célébrer la victoire, ils n'ont été gênés à ce point par la pensée des morts. C'est pourtant cette fois-ci une bien grande victoire; la plus grande en un sens, qu'il y ait jamais eu. Oui, mais c'est peut-être aussi qu'il n'y a jamais eu tant de morts. Dans cette mathématique de la folie humaine, il doit exister des limites qu'il est imprudent de franchir. Les proportions se disloquent. Le poids des morts grandit plus vite que la fierté des vainqueurs. Le tas des morts grimpe plus vite que le trophée. La victoire a beau grandir, elle ne réussit plus à rattraper les morts ${ }^{14} »$.

7 La décision d'ériger un monument est donc généralement prise dans le courant de l'année 1919 et, pendant plusieurs années, le culte des morts va s'organiser autour de l'avancement des travaux, jusqu'au moment crucial de l'inauguration.

Qu'en est-il du financement de ces entreprises ? Habitués à bénéficier des services rendus par la direction des beaux-arts pour l'embellissement de leurs locaux, plusieurs établissements se tournèrent spontanément vers cette administration pour lui demander de prendre à sa charge l'ensemble des travaux. Les écoles pouvaient en outre se prévaloir du sang versé pour la cause commune, ce que ne manqua pas de faire Ernest Lavisse :

« Nous ne demandons pas seulement l'autorisation nécessaire pour entreprendre la construction. Vous savez quelle a été l'hécatombe des normaliens, presque la moitié de ceux qui sont partis en 1914 ne sont pas revenus ; après un tel sacrifice, peut- 
être l'État jugera-t-il que c'est à lui d'inscrire sur les murs d'un édifice qui lui appartient les noms de ceux qui ont donné leur vie, et d'y commémorer l'ardente réponse de toute l'École Normale à l'appel du devoir ${ }^{15}$. "

9 Mais la rue de Valois se dérobe à cette proposition, mettant en avant la modicité des crédits qui lui sont alloués en ces années difficiles, et probablement désireuse de ne pas créer un précédent qui ouvrirait la voie à de trop nombreuses demandes : elle propose de verser une simple subvention, une fois connu le montant total des travaux engagés ${ }^{16}$. En cette matière comme en bien d'autres, le cas de l'École normale est emblématique : la commande publique étant écartée, reste la souscription; rue d'Ulm comme dans les autres établissements ce mode de financement contribuera à faire des monuments aux morts l'expression directe des souhaits et désirs de la communauté, sans que les pouvoirs publics ne viennent imposer leurs conceptions ${ }^{17}$. Ces souscriptions, lorsque le détail en est connu, nous renseignent utilement sur les différentes strates de ces communautés humaines, elles permettent d'entrevoir, au-delà du cercle des administrateurs, des professeurs et des élèves, quels sont les acteurs de la société qui ont partie liée avec la communauté et tiennent à elle de quelque manière, ce que l'examen de la souscription de Normale montre bien ${ }^{18}$. Deux remarques s'imposent d'emblée. D'une part, l'importance des fonds recueillis. Nous ignorons le montant des souscriptions réunies dans le cas de l'École Polytechnique ou dans celui de l'École Centrale, mais la taille des œuvres est comparable à celle de monuments mis en place dans des villes de moyenne importance, ayant rang de préfecture, ce qui permet d'évaluer leur coût entre 50 et 100000 francs pour Polytechnique, sans doute bien plus pour Centrale ${ }^{19}$. Chez les normaliens, la première souscription rassemble 28026 francs, la seconde 10687 francs $^{20}$. D'autre part, les structures mises en place en vue de la collecte des fonds. Il peut arriver que l'administration de l'établissement se charge personnellement de l'affaire, mais ce n'est pas le cas le plus fréquent ${ }^{21}$. Généralement, un comité formé par d'anciens élèves, distinct de l'administration, s'occupe de toute l'entreprise : décidément, c'est bien le tissu humain de la communauté qui prend soin d'honorer les disparus, sans abandonner ce devoir à l'ossature administrative ou aux pouvoirs publics. Revenons sur l'exemple de l'École Normale Supérieure, puisque le détail de cette souscription est bien connu ${ }^{22}$. Dans un premier cercle de souscripteurs se trouvent les normaliens, leurs familles, les administrateurs de l'école: c'est la masse de leurs dons, bien souvent modestes, qui constitue l'assise de la souscription. Le nombre important des participants témoigne de la cohésion du groupe. Ainsi, Ernest Lavisse donne 200 francs, dès 1919. Certaines institutions, certaines entreprises, quand elles entretiennent des liens avec l'école, soit qu'elles emploient des anciens élèves, soit qu'elles soient liées au monde intellectuel et universitaire parisien, apparaissent également en bonne place: les éditions BergerLevrault et Armand-Colin, voisines de l'école, versent toutes deux 500 francs $^{23}$. Les autres institutions universitaires participent à l'opération, en particulier lorsqu'elles sont proches de la rue d'Ulm, puisque les normaliennes de Fontenay-aux-Roses apportent 1000 francs. En dehors de cette sphère d'influence directe de l'école, on rencontre les dons provenant de mécènes; leur nombre, leur qualité et le montant de leur contribution attestent du prestige des normaliens dans la société. A. de Rothschild et Albert Kahn donnent chacun 1000 francs, il en va de même pour la marquise Arconati-Visconti ${ }^{24}$. Enfin, si les contributions de la direction des beaux-arts ne sont guère importantes ${ }^{25}$, il faut mentionner, a contrario, la générosité de la Ville de Paris: le conseil municipal accorde d'abord 2000 francs, puis 3000 francs supplémentaires, pour hâter l'achèvement des travaux. Enfin, le Président de la République a versé 300 francs. Une souscription 
limitée aux membres de la communauté n'est pas nécessairement l'indice d'un moindre prestige. Lorsque le caractère familial de l'hommage est revendiqué, il peut témoigner de la grande cohésion du groupe, et ce souci d'intimité est présenté comme un des traits distinctifs de ses membres: le mode de financement devient alors une forme de représentation de l'institution. Les ouvrages consacrés à l'École Normale Supérieure de Saint-Cloud ${ }^{26}$ insistent volontiers sur cet aspect, et l'on n'est pas surpris d'en trouver l'écho dans le récit de la souscription:

«Dès le moment où il a été possible de songer à l'accomplissement de ce devoir, l'association en a assumé la charge comme un privilège (...). Honorée et aimée comme elle l'est dans le monde de l'enseignement primaire, l'École pouvait recueillir [pour] glorifier ses morts de la guerre, de quoi ériger devant sa façade un monument public de pierre et de marbre. Mais les camarades ont pensé, et nous avons pensé avec eux, qu'un hommage fraternel et plus intime était mieux en harmonie avec la tradition et l'esprit de cette école, qui s'est toujours considérée comme une famille, et qui a toujours mené sans bruit son utile labeur ${ }^{27}$. "

Mais, lorsque l'orateur évoque les souscripteurs qui se seraient probablement manifestés, nous retrouvons, avec un éventail social sensiblement différent, une configuration identique à celle de la souscription des ulmiens ; autour des membres de la communauté se regroupent tous ceux qui lui sont liés par leur formation ou leur profession :

«Si l'on avait voulu ouvrir à travers l'enseignement primaire une souscription pour l'érection du monument aux morts (...), de toutes parts, les offrandes auraient afflué : combien d'instituteurs aux cheveux gris se souviennent affectueusement des écoliers d'élite dont ils ont reconnu la vocation pour l'étude et l'enseignement et qu'ils ont acheminés vers cette école ! Combien, parmi les instituteurs des générations moins avancées en âge, se souviennent avec gratitude des professeurs de l'École normale, anciens de Saint-cloud, dont ils ont reçu l'enseignement ${ }^{28}$ ! "

11 Par leur mode de financement, les monuments aux morts des établissements d'enseignement supérieur révèlent des communautés étroitement soudées et attestent de l'existence d'un culte des disparus qui prend des formes originales, différentes de celles suggérées par les monuments communaux ou nationaux. Il peut arriver que le choix des artistes participe de cet esprit. Dans la plupart des cas, la communauté ne possède pas en son sein de membre capable d'exécuter une œuvre répondant aux souhaits de ses confrères: il faut donc faire appel à un artiste extérieur, qui travaillera d'après les indications des membres du comité, et ce dernier examinera les projets successifs. Dans le dossier du monument aux morts des normaliens, on retrouve des photographies présentant les différentes étapes du projet de Paul Landowski ${ }^{29}$. Le comité choisit l'artiste en partie en fonction de la somme réunie ; parmi les personnalités retenues, certaines ont exécuté auparavant ou par la suite plusieurs monuments commémoratifs de la Grande Guerre: Victor Ségoffin, Paul Landowski (monument aux morts de la faculté mixte de médecine et de pharmacie de Bordeaux, monument commémoratif de la bataille de Champagne), tandis que le haut-relief de l'École vétérinaire de Maisons-Alfort est identique au monument de l'École de cavalerie de Saumur (fig. $\mathbf{n}^{\circ} \mathbf{1}$ ). En revanche, dans les écoles d'art, le concepteur et le sculpteur sont issus de l'institution. Enfin, à l'École centrale des arts et manufactures, c'est un centralien, l'architecte Leprince-Ringuet, qui conçut les plans de l'arc de triomphe de l'école et assigna au sculpteur Marcel Gaumont les emplacements à décorer. (fig. $\mathbf{n}^{\circ} \mathbf{2}$ ) 
Figure 1

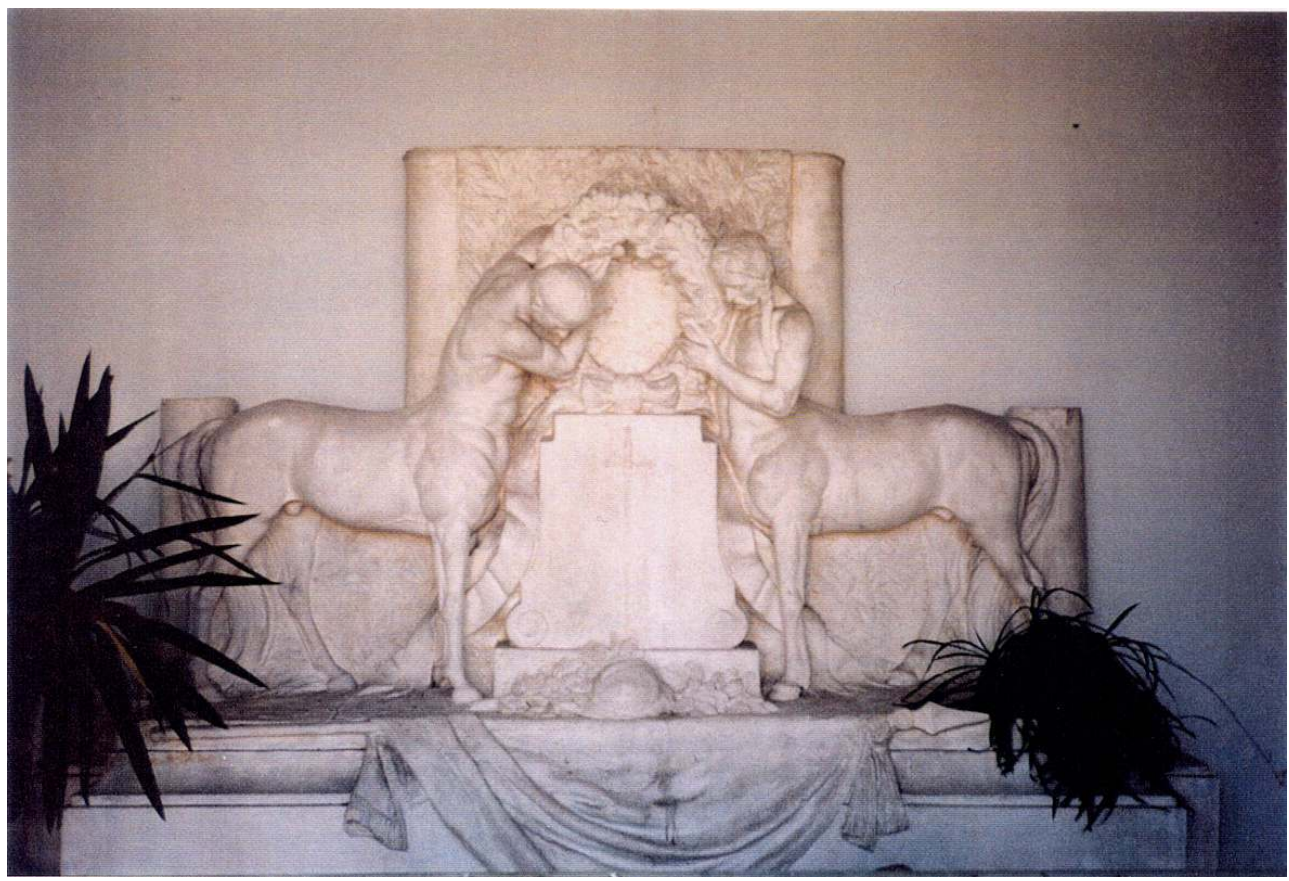

J. Froment-Meurice, monument aux morts de l'École nationale vétérinaire de Maisons-Alfort, s.d. Phot. Christian Hottin, 1995.

Figure 2

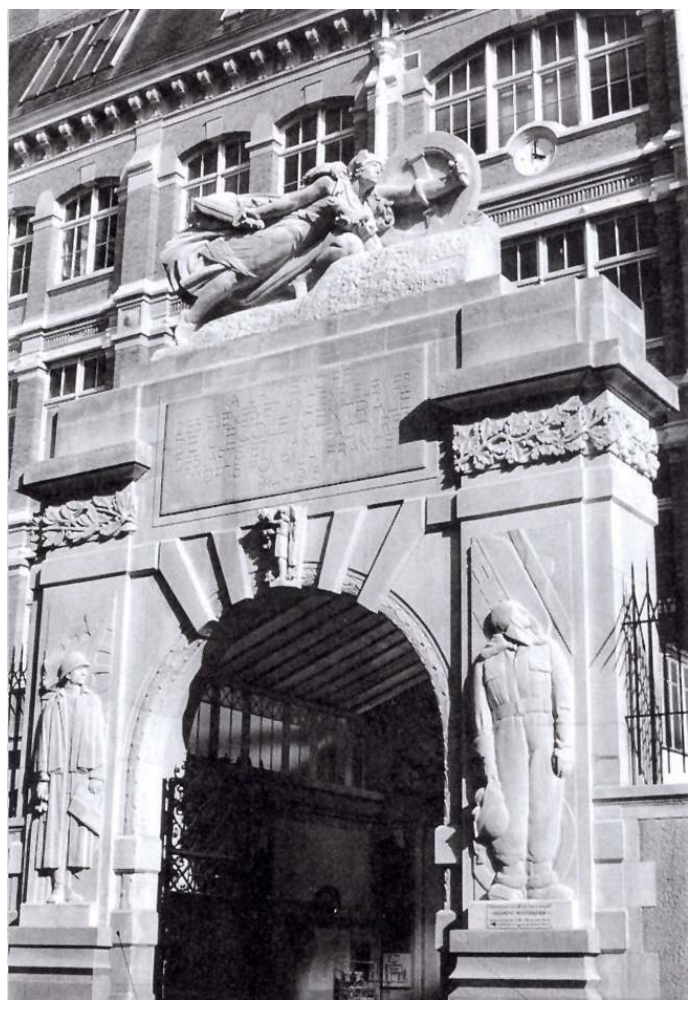

M. Gaumont et P. Leprince-Ringuet, monument aux morts de l'École centrale des arts et manufactures (Paris), 1923.

PHOT. PIERRE HAgARD, 1996. 
Moment grave, la cérémonie d'inauguration est caractérisée par un important décorum (gerbes et orchestre) et par de nombreux discours. Certaines sont connues à travers les comptes rendus publiés dans L'Illustration, puisque, dans les années suivant la guerre, cette revue possédait une rubrique spécialement consacrée aux monuments aux morts... Ces événements paraissent avoir marqué les esprits, à tel point qu'il en est fait mention dans les ouvrages consacrés à la vie des écoles ${ }^{30}$. Chaque cérémonie comporte en fait deux versants bien distincts et dotés de significations particulières. Un premier échange de discours, entre le président du comité chargé du monument et le directeur de l'établissement, est en fait la remise du monument à l'école par les membres de la communauté. Il s'agit, symboliquement, d'un hommage de la communauté fait à ellemême, d'une auto-célébration. En cette occasion, la communauté reconnaît ses mérites, la grandeur du sacrifice consenti, mais, d'une manière plus abstraite et intemporelle elle affirme sa dualité et la richesse de sa diversité. Les anciens élèves honorent la maison qui les a formés et dont ils tiennent leurs mérites, cette dernière salue la gloire de ses fils qui ont été dignes d'elle: à aucun moment, sans doute, l'osmose entre l'enveloppe administrative de l'institution et le tissu humain de la communauté n'est aussi totale. Ce mouvement d'échange et d'hommage réciproque est bien rendu par le texte de ce compte rendu, concernant le monument des centraliens :

«Dimanche, sous la voûte d'entrée, des gerbes et des couronnes avaient été amoncelées par de pieuses mains. M. Henri Garnier, président de l'association amicale des anciens élèves, a remis au directeur, $\mathrm{M}$. Léon Guillet, le monument. Tous deux, dans les émouvants discours qu'ils échangèrent, évoquèrent la gloire de l'école et ses deuils; et M. Gaston Vidal, en de hautes paroles, dit le noble enseignement qui se dégageait de tant de sacrifices ${ }^{31}$."

Cet hommage de l'institution à ses disparus, acte fondamental qui crée le monument en tant que centre du culte des morts, prend place dans une cérémonie plus ouverte, pleinement publique, au cours de laquelle toute l'école, représentée par sa structure bicéphale (continuité du corps administratif et communauté vivante des membres), reçoit l'hommage de la Nation. En effet, les corps constitués, les autres écoles et l'armée, l'église et les élus sont là. Qu'ils parlent ou non, leur présence constitue en soi un hommage à l'établissement. Si le Président de la République est présent et prononce un discours, comme ce fut le cas lors des séances de rentrée de Polytechnique et de Normale, le salut aux défunts débouche naturellement sur le rappel du rôle tenu par les membres de l'institution au cours du conflit, et, de là, sur la reconnaissance de la Nation pour les services rendus et les missions accomplies en tous temps par l'école. Ce second versant de la cérémonie revêt donc une importance capitale pour le groupe : après avoir affirmé son sentiment de communauté, il voit sa spécificité reconnue par la Nation. À travers les morts, son action au sein du pays est exaltée, mais son originalité est perçue et même magnifiée. L'inauguration est donc pour le groupe une occasion exceptionnelle de se poser, sans pour autant s'opposer. Une cérémonie comparable à l'inauguration d'un monument a été représentée par une peinture, transformant ainsi la représentation éphémère en une représentation permanente et emblématique du rayonnement de l'institution: La Remise de la Croix de la Légion d'Honneur à l'École Centrale des Arts et Manufactures, œuvre de Paul-Albert Laurens ${ }^{32}$. L'artiste a montré les différents représentants des instances dirigeantes de la Nation groupés dans la cour de l'école, tandis que, sur une estrade, Léon Guillet, entouré des administrateurs et professeurs de Centrale, des représentants de l'amicale des anciens élèves et des majors des trois dernières promotions, reçoit la Croix des mains du président ; rayonnant dans son habit 
de membre de l'Institut, le directeur de Centrale domine largement la sombre jaquette de ce dernier ! (fig. $\mathbf{n}^{\circ} \mathbf{3}$ )

Figure 3

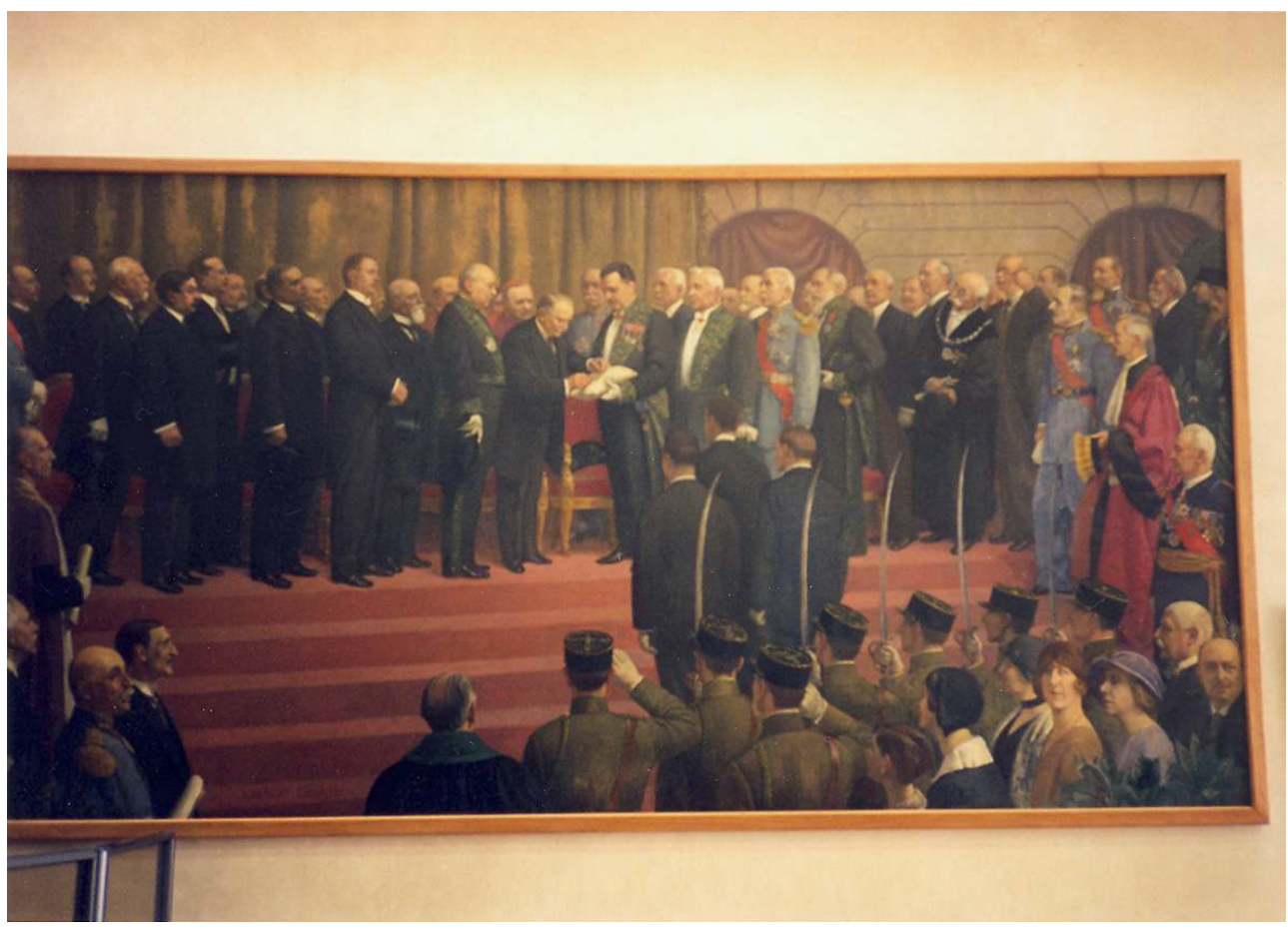

P.A. LAURENS, REMISE dE LA CROIX DE LA LÉgION D'HONNEUR À L'ÉCOLE CENTRALE DES ARTS ET MANUFACTURES, 1929.

PHOT. CHRISTIAN HOTTIN 1995.

La genèse des monuments, de la souscription à l'inauguration, nous laisse voir que le culte des morts de la Grande Guerre est, au sein des établissements d'enseignement supérieur, une expression des rapports entretenus par ces communautés avec la Nation, qu'il s'agisse des pouvoirs publics, dans un premier temps, ou des représentants politiques, après l'achèvement de l'œuvre. Si ce culte s'intègre, comme nous l'avons dit en introduction, dans un vaste mouvement qui plonge alors tout le pays dans un climat lugubre, il n'en recèle pas moins des particularités, signes précieux pour comprendre les systèmes de représentation de ces communautés. Cette ambiguïté se retrouve dans les monuments eux-mêmes.

\section{Les monuments, expression du culte des morts et représentation de l'institution}

Parmi les monuments aux morts des établissements d'enseignement supérieur, il en est qui ne se distinguent en rien des modèles les plus répandus ; c'est le cas, par exemple, du poilu installé dans la cour du mûrier de l'École des beaux-arts : sanglé dans son uniforme et tenant son fusil, il semble monter la garde dans une attitude résolue. Il ne s'agit sans doute pas d'un poilu triomphant, pour reprendre l'expression employée par Antoine Prost, mais on ne relève sur cette statue aucun caractère évoquant la spécificité de la communauté qu'elle est censée honorer. (fig. $\mathbf{n}^{\circ} 4$ ) 
Figure 4

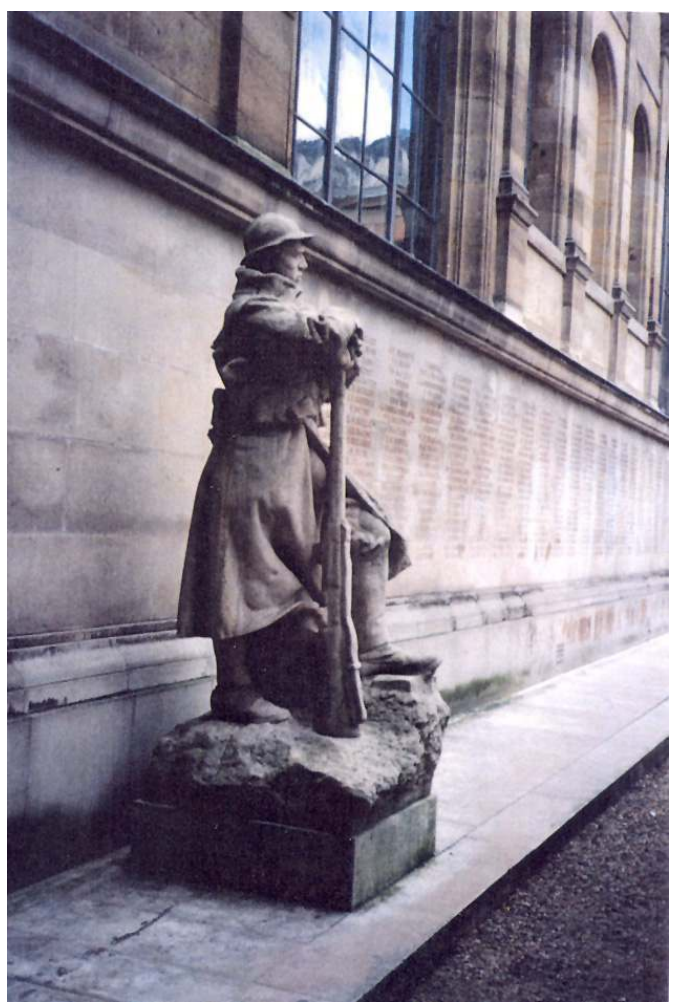

J. Boucher, monument aux morts de l'École nationale supérieure des Beaux-arts (Paris), 1924. PHOT. CHRISTIAN HOTTIN, 1995.

En fait, les artistes chargés de concevoir les monuments ont bien souvent insisté sur une des particularités des défunts : ayant à œuvrer pour des institutions d'enseignement, ils ont plus volontiers figuré des individus jeunes. Cette représentation ne correspond que partiellement à la réalité, puisque, au sein des universités et des grandes écoles, se trouvaient des mobilisés issus de toutes les classes d'âge. Il est en revanche exact que les jeunes générations furent particulièrement frappées. Ce choix des artistes donne donc aux poilus de nos monuments une physionomie quelque peu différente de celle des soldats accompagnant les monuments municipaux: au type inspiré du paysan français (portant une ample moustache) ${ }^{33}$, on préfère celui du jeune homme, dont le fin visage est souvent idéalisé. Le monument élevé aux étudiants de toutes les disciplines morts pour la France (un bas-relief apposé dans la cour de la Faculté de droit), (fig. $\mathbf{n}^{\circ}{ }^{\circ}$ ) la figure de l'auxiliaire de pharmacie sculpté par Coutheilas, le transi de la Faculté des sciences, et, bien sûr, l'éphèbe couché par Landowski devant la liste des normaliens (sans oublier les personnages du monument aux morts de la Faculté mixte de médecine et de pharmacie de Bordeaux, autre œuvre de Landowski) (fig. $\mathbf{n}^{\circ} \mathbf{6}$ ) sont assez représentatifs de ce souci. L'accent porté sur l'âge des victimes ne traduit pas seulement le désir de faire correspondre le monument avec la réalité ; ce qui compte le plus, bien entendu, c'est l'émotion particulièrement intense qui naît de ce rapprochement: ces figures de la jeunesse foudroyée traduisent l'horreur qu'inspire la mort d'êtres en plein développement de leur personnalité, délicats, peu préparés à la guerre et promis à une existence brillante. Au chagrin d'une vie perdue, on voit s'ajouter le scandale d'une 
intelligence détruite. Les critiques d'art ont rendu compte de la puissance pathétique de ces œuvres :

«Saisissant et puissant morceau [que cette sculpture], dont le modèle délicat évoque les jeunes penseurs au corps pétri d'intelligence. Émouvante personnification dont le geste harmonieux enveloppe tout le monument d'une sorte de poésie héroïque ${ }^{34}$.»

Figure 5

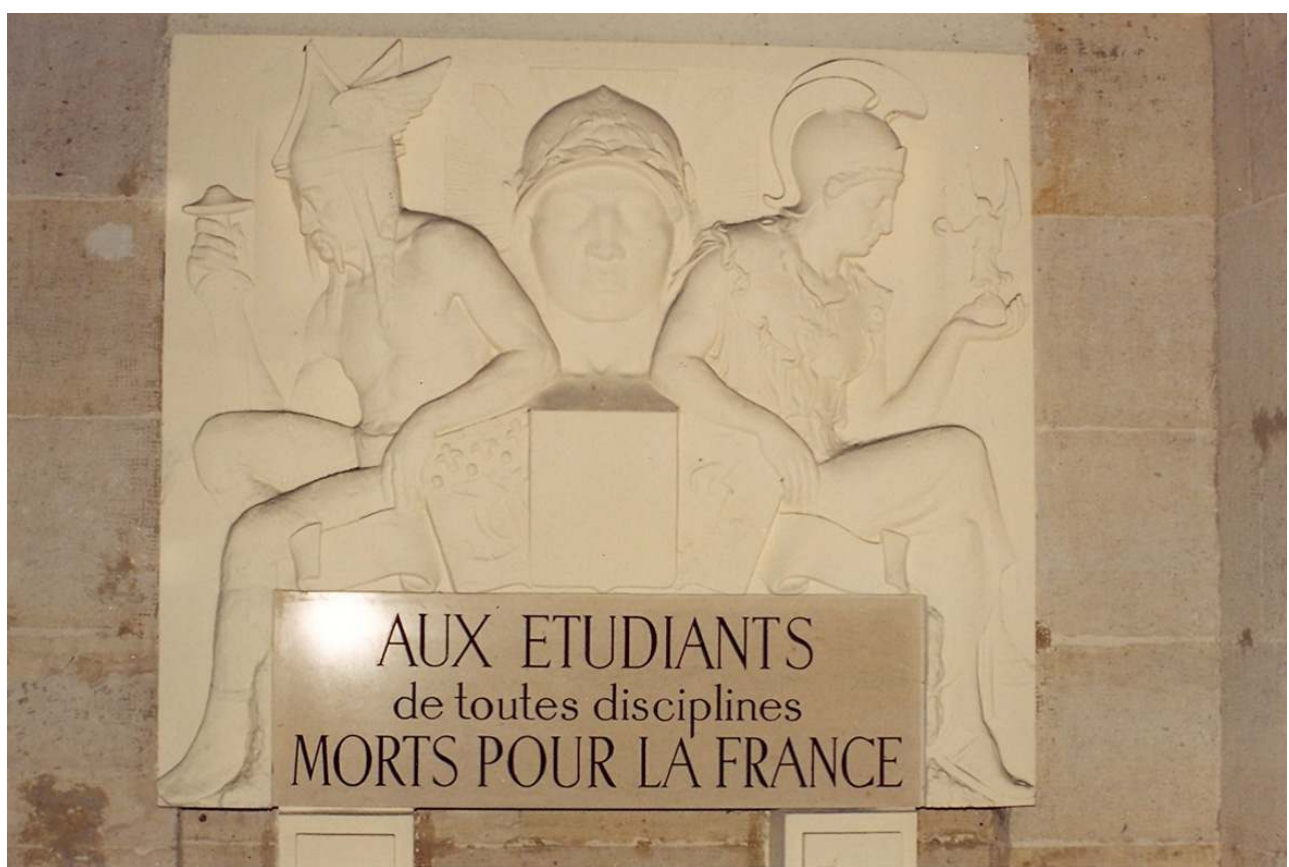

JOANNY DURAND, MONUMENT AUX ÉTUDIANTS DE TOUTES LES DISCIPLINES MORTS POUR LA FRANCE (PARIS, FACULTÉ DE DROIT DU PANTHÉON), CA. 1920.

PHOT. CHRISTIAN HOTTIN, 1995. 
Figure 6

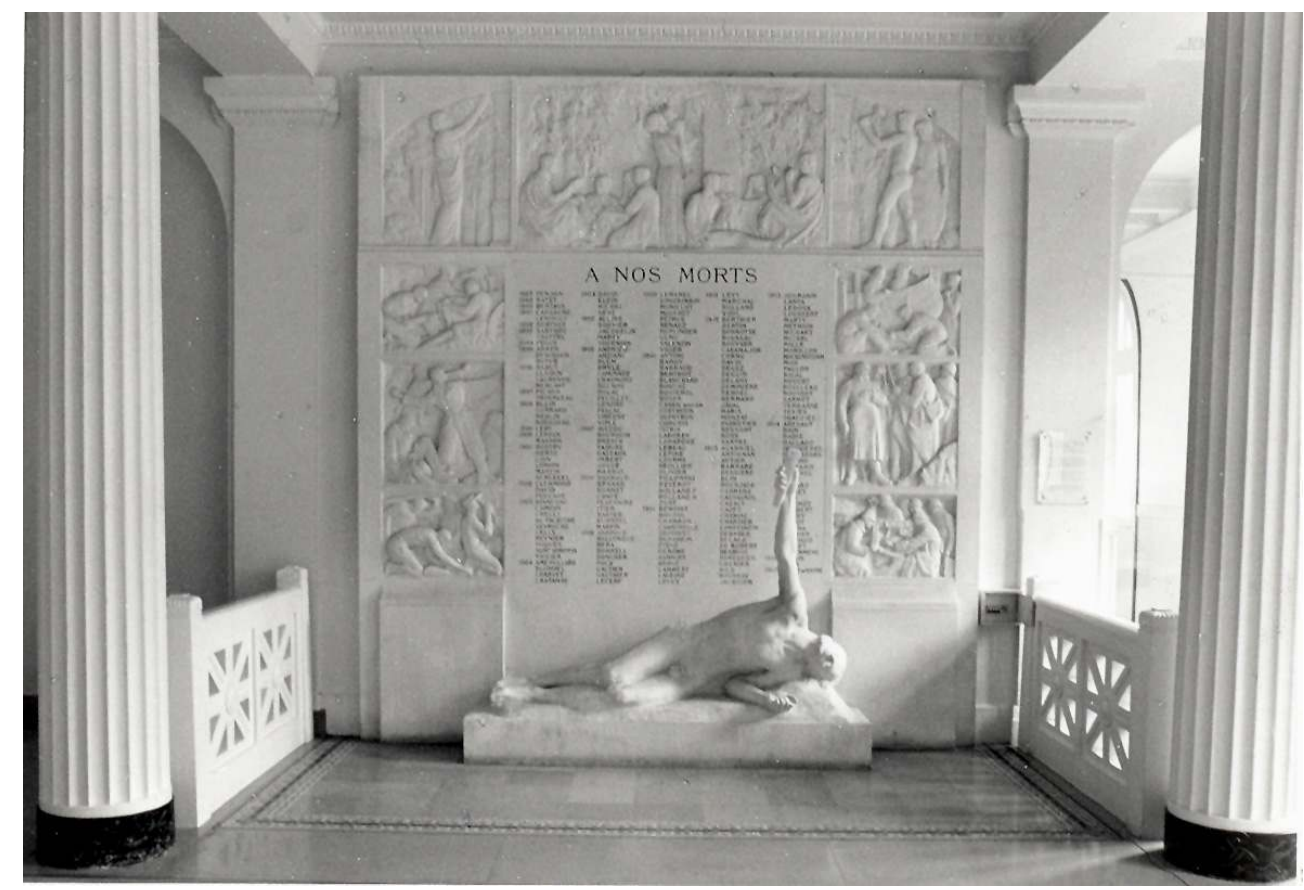

PAUL LANDOWSKI, MONUMENT AUX MORTS DE L'ÉCOLE NORMALE SUPÉRIEURE (PARIS), 1921.

PHOT. PHILIPPE REIX, 1995.

17 Ces jeunes figures sont autant de traductions sculpturales d'un malaise que nous retrouvons, sublimé par l'art oratoire, dans plusieurs discours prononcés à la même époque : comment admettre la mort, dans des conditions souvent horribles, d'individus élevés dans l'amour de la raison et qui n'avaient pas encore eu la possibilité de donner pleinement leur mesure ? Les tentatives de justification ne paraissent guère convaincantes:

«Si quelque chose peut nous consoler de leur départ, c'est la certitude que, tombés en pleine jeunesse, ils ont rempli leur tâche humaine. Ils ont versé dans une vie courte et sublime la force capable d'animer une longue existence, ils ont condensé dans une brève série d'actions fécondes l'essence de leur âme $(. . .)^{35}$. » 
Figure 7

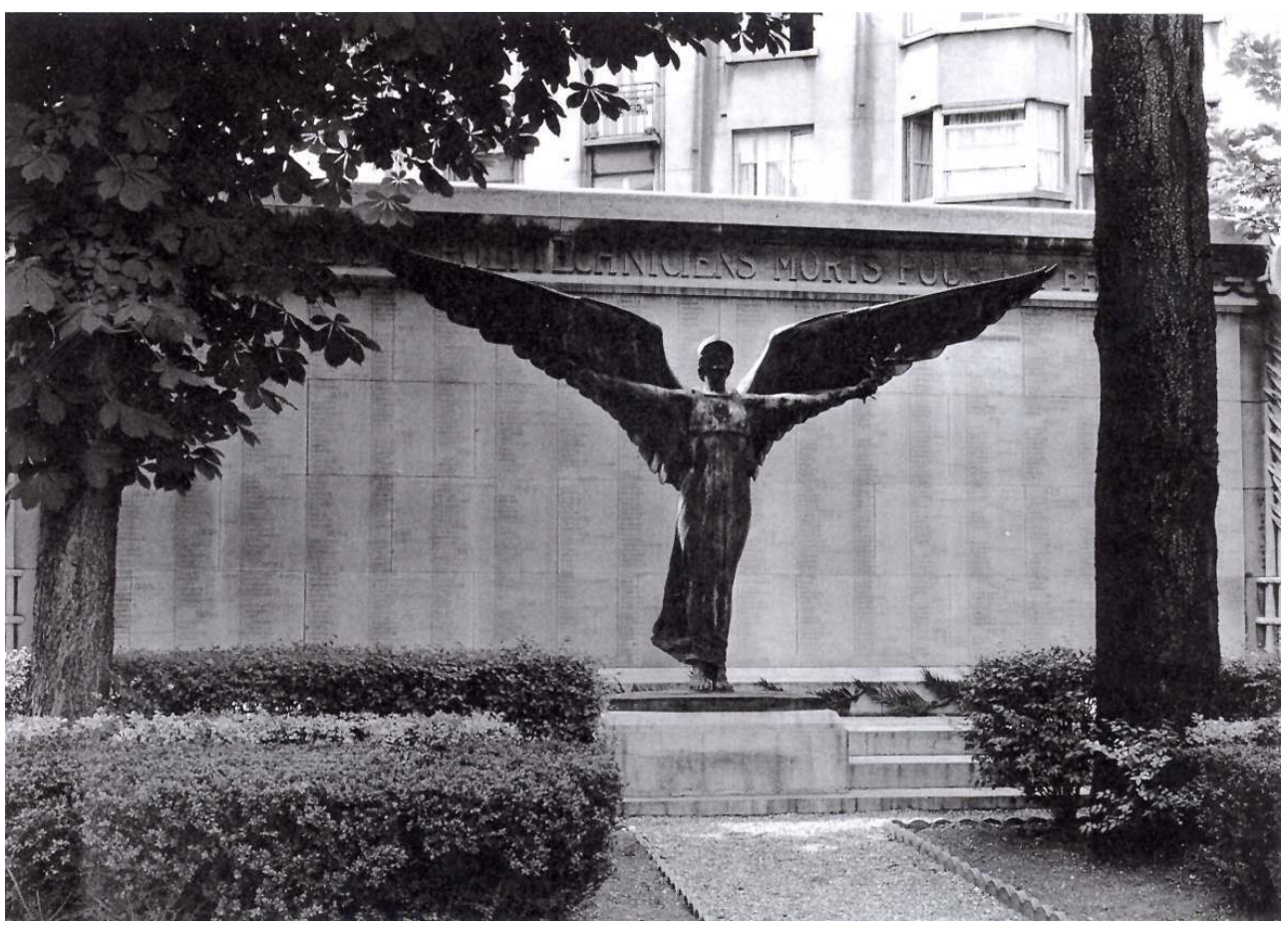

VICTOR SÉgOffin, MONUMENT AUX MORTS DE L'ÉCOLE POLYTECHNIQUE (PARIS), 1924.

PHOT. ÉTABLISSEMENT PHOTOgRAPHIQUe ET CINÉMATOgRAPHIQUE DES ARMÉES, S.D.

Figure 8

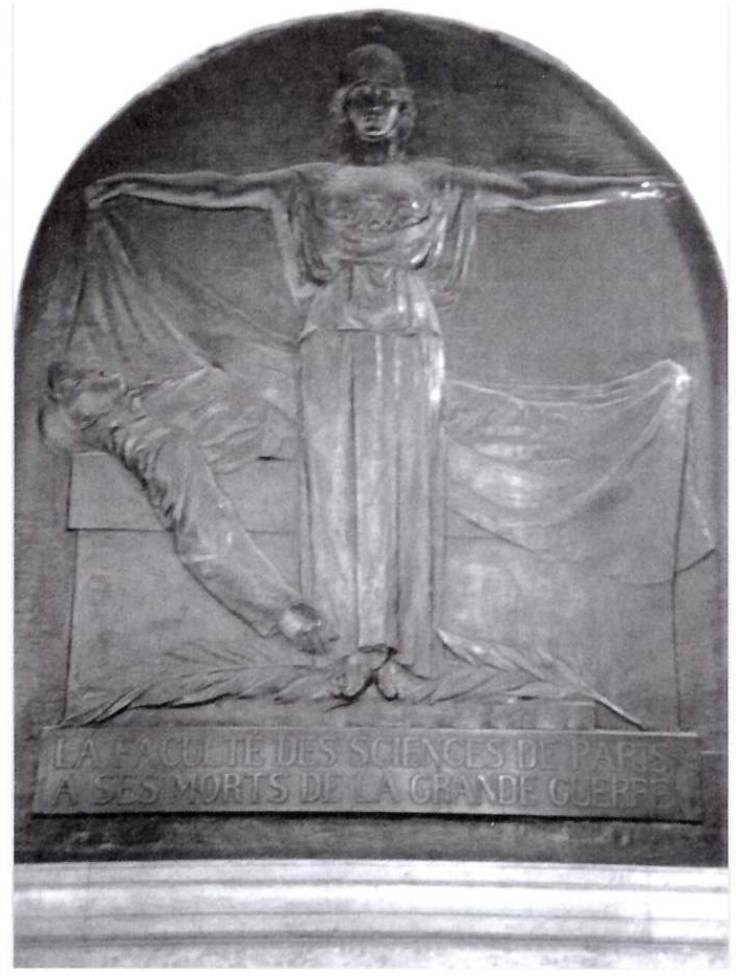

F. BENNETEAU, MONUMENT AUX MORTS DE LA FACULTÉ DES SCIENCES (PARIS, SORBONNE), 1923.

PHOT. CHRISTIAN HOTTIN, 1995. 
Comme on le voit, ce premier élément de spécificité ne distingue que très marginalement les monuments aux morts des établissements d'enseignement supérieur des autres. Il en est un second, relevant également en grande partie du libre choix de l'artiste, qui introduit une véritable forme de modulation entre les différentes institutions : les figures d'accompagnement. Ces personnages, le plus souvent de sexe féminin, qui se tiennent près des représentations des défunts ont toujours été fort répandus dans l'art funéraire. Quand il s'agit d'allégories assorties d'objets indiquant leur signification, ces sculptures permettent de caractériser le personnage principal : elles dévoilent sa dimension sociale, tandis que le buste restitue son apparence physique ${ }^{36}$. Dans les monuments aux morts des communes, lorsque cette figure d'accompagnement n'est pas la Victoire ou la France, il est fréquent qu'elle renvoie à la cellule familiale (veuve et orphelin, mère éplorée). Antoine Prost donne comme illustrations de ce type de monuments ceux de Ganges et Suippes $^{37}$. De même, le monument élevé place Hubert-Monmarché, dans le $\mathrm{XV}^{\mathrm{e}}$ arrondissement de Paris, montre le soldat entre une femme en grand deuil accompagnée d'un garçonnet et un groupe de combattants représentant toutes les époques de l'histoire de France. Le mort est symboliquement placé au point de rencontre de la destinée individuelle et de la geste nationale. Or, dans les monuments aux morts des universités et des grandes écoles, ces figures cèdent la place à d'autres, évocatrices des enseignements dispensés au sein des institutions ou représentatives de vertus caractéristiques de la carrière embrassée à la sortie de l'école. (fig. $\mathbf{n}^{\circ} 7$ ) La victoire ailée et casquée imaginée par Victor Ségoffin pour le monument des polytechniciens évoque plus particulièrement la vocation initiale et le statut militaire de l'école. À la Faculté des sciences, la figure de Minerve symbolise aussi bien la guerre que l'idée de connaissance (fig. $\left.\mathbf{n}^{\circ} \mathbf{8}\right)$, tandis que la femme assise aux pieds du pharmacien des armées agonisant (fig. $\left.\mathbf{n}^{\circ} \mathbf{9}\right)$ (fig. $\left.\mathbf{n}^{\circ} \mathbf{1 0}\right)$ est ouvertement présentée comme une figure de la science interrompant ses lectures (elle tient un livre en main) pour méditer sur la mort ${ }^{38}$. La figure de la science est à cette occasion rapprochée de celle de la mère, dont les pharmaciens deviennent en quelque sorte les enfants. 
Figure 9

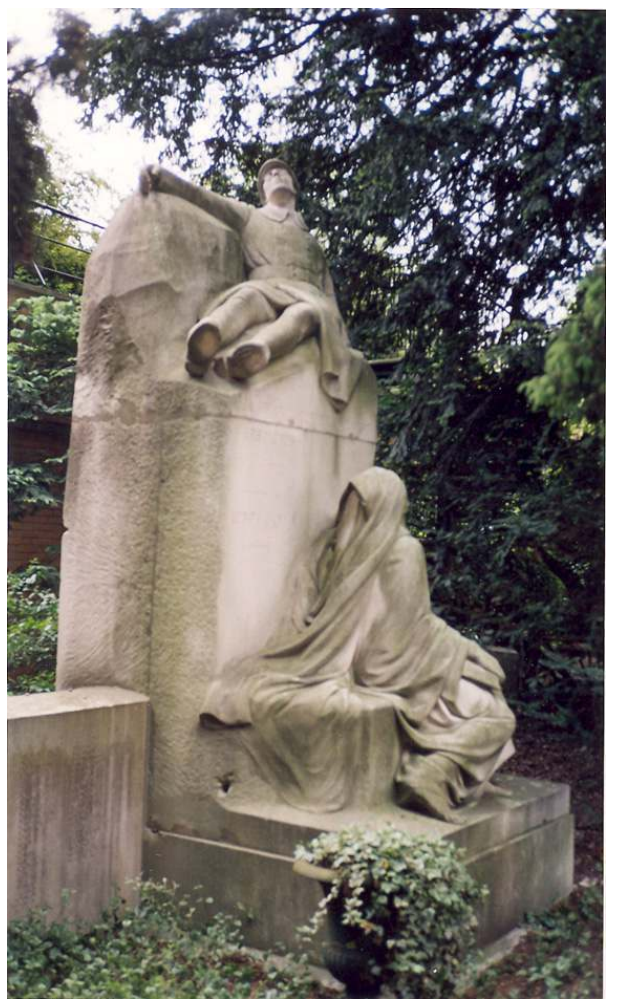

H. COUTHEILAS, MONUMENT AUX MORTS de L'ÉCOLE SUPÉRIEURE de PHARMACIE (PARIS), 1926. PHOT. CHRISTIAN HOTTIN, 1995. 
Figure 10

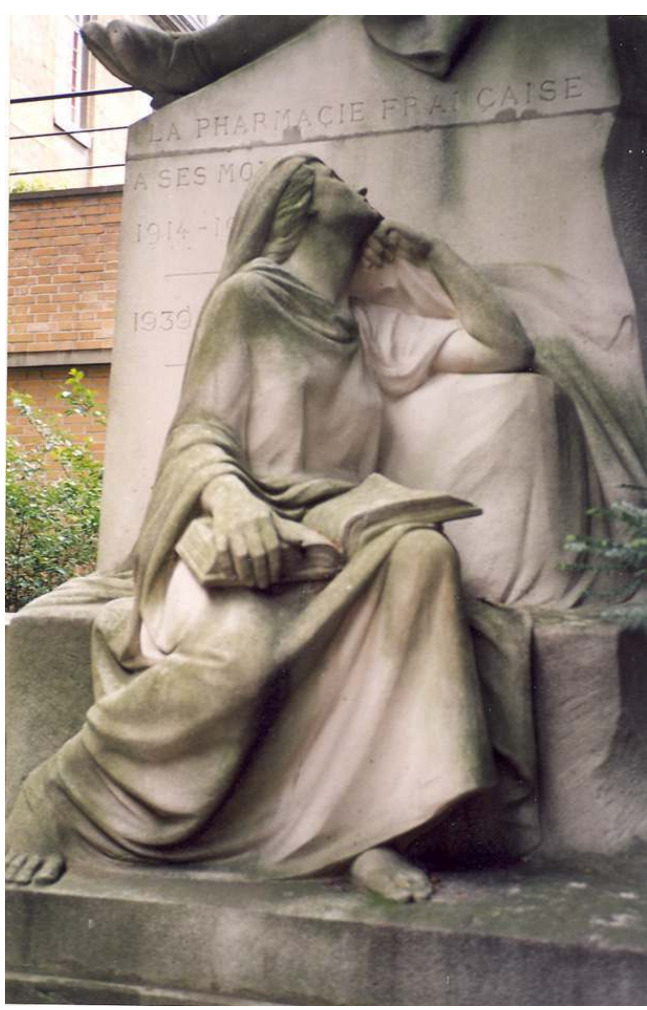

H. COUTHEILAS, MONUMENT AUX MORTS De L'ÉCOLE SUPÉRIEURE De PHARMACIE (PARIS), 1926. PHOT. CHRISTIAN HOTTIN, 1995.

Il en va de même à l'Institut agronomique, dont le monument est extérieurement très proche de celui de la commune de Suippes : une femme se recueille devant une tombe, elle porte une gerbe de blé et de fleurs. Mais il y a loin de la paysanne champenoise de Suippes à la jeune fille soigneusement coiffée de l'I.N.A., (fig. $\mathbf{n}^{\circ}{ }^{\circ 11}$ ) puisque cette dernière est qualifiée de Cérès symbolique ${ }^{39}$ et paraît bien être une figure de l'agronomie rendant hommage, ici encore, à ses enfants. Quant aux bas-reliefs qui accompagnent le monument ils évoquent le monde rural: ruches, vergers et rossignols. À l'École vétérinaire de Maisons-Alfort, ce sont des centaures (l'un portant des nattes gauloises et l'autre une courte coiffure romaine) qui déposent une couronne sur le tombeau. 


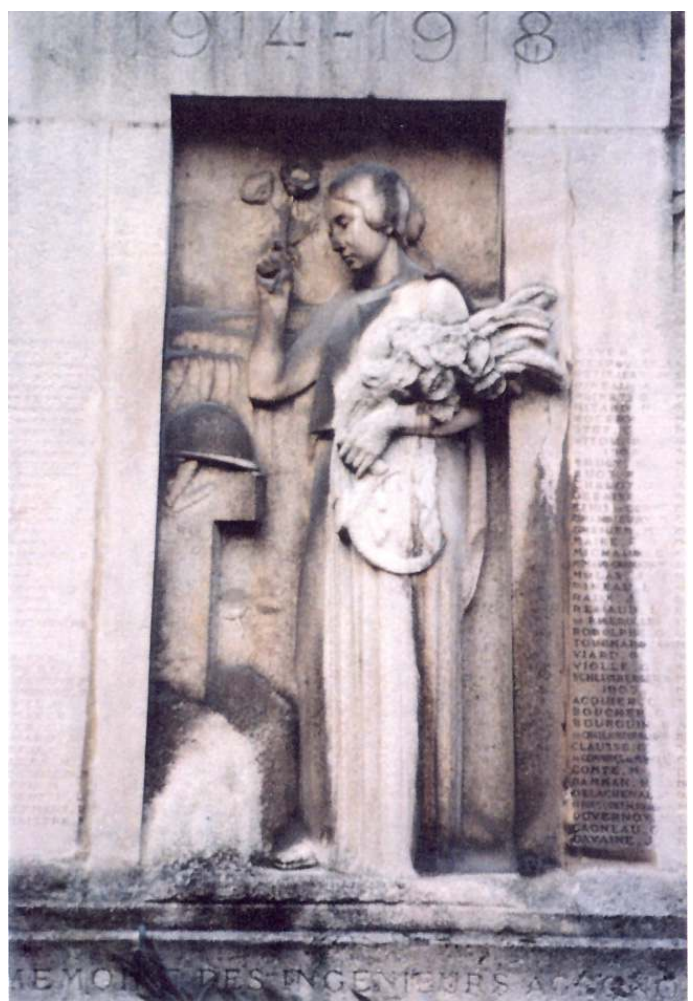

L. BRASSEUR, MONUMENT AUX MORTS DE L'INSTITUT NATIONAL AgRONOMIQUE (PARIS), 1921.

PHOT. CHRISTIAN HOTTIN, 1995.

Mais les monuments aux morts des établissements d'enseignement supérieur ne se distinguent pas seulement des autres par l'allure juvénile des combattants ou la signification des figures d'accompagnement: en eux, nous retrouvons le dialogue de l'institution avec la Nation, amorcé lors de la construction et de l'inauguration. Plus exactement, c'est à travers leur forme que la communauté laisse entrevoir les éléments de représentation qui fondent sa spécificité, ils sont l'occasion d'affirmer son originalité au sein du pays. Un premier caractère distinctif nous livre leur conception particulière du temps et de la société. À l'ordre alphabétique, le plus répandu dans les listes de défunts ${ }^{40}$, nombre de grandes écoles substituent la répartition par année de promotion : École des ponts et chaussées, École des mines, Polytechnique, Normale, Centrale, etc. Lorsque l'ordre alphabétique est choisi, on fait suivre le nom de l'année de promotion. Cette date constitue un lien entre les morts et les vivants, entre les anciens et les nouveaux, elle permet aux seuls membres de la communauté de retrouver un camarade, grâce à cette donnée souvent ignorée du profane. Le temps universel (1914-1918, éventuellement raffiné par le détail des années de lutte) est remplacé par le temps particulier du groupe : Charles Péguy, tombé en 1914, est avec ceux de sa promotion, celle des Lettres de 1894. Ainsi, les anciens condisciples sont symboliquement réunis dans la mort. On retrouve la trace de cette perception originale du temps dans les cérémonies commémoratives de l'École centrale. En effet, Chaque année, le 3 novembre, le directeur et les élèves viennent déposer des fleurs et des palmes sur les grandes plaques de marbre où sont inscrits en lettres d'or les noms des cinq-cent cinquante centraux tués à la guerre ${ }^{41}$. Ce n'est pas la proximité du jour des morts qui explique cette date, mais le souvenir de la naissance de l'institution, puisque les premiers cours de l'École centrale des arts et manufactures se tinrent le 3 novembre 1829. 
Cette mise en scène d'une chronologie propre aux institutions se manifeste dans les monuments aux morts, mais elle est également un trait essentiel des systèmes de représentation universitaires pris dans leur ensemble: célébration des origines, culte rendu aux fondateurs, insertion d'un temps cyclique original dans le temps de la société (celui de la rentrée scolaire, marqué symboliquement par les rites initiatiques), etc. Ces listes de défunts nous restituent parfois les hiérarchies qui structurent les communautés. Ce n'est pas le cas dans les monuments élevés par les communes, puisque moins de $4 \%$ de ces derniers font apparaitre les hiérarchies militaires. L'ordre alphabétique donne un aspect égalitaire aux monuments. Les officiers n'y apparaissent pas, toute hiérarchie est gommée $e^{42}$. Cette hiérarchie n'apparaît jamais sur nos monuments, en revanche celle des établissements s'y montre de plusieurs manières. Une première distinction est généralement opérée entre les élèves et le corps enseignant. Lorsque les membres de ce dernier ne bénéficient pas d'une plaque particulière, ils sont souvent cités en tête et leurs noms apparaissent en plus gros caractères : c'est le cas à l'École pratique des hautes études, où les directeurs d'études occupent le haut de la plaque, tandis que les noms des élèves s'alignent au-dessous, en lettres beaucoup plus minces. À partir de cette distinction de base, il existe de nombreux raffinements possibles : chez les juristes, les professeurs agrégés sont distingués des simples chargés de conférences. La structure particulière des écoles des mines et des ponts et chaussées se prête fort à de telles distinctions: les corpsards issus de l'École polytechnique et sortis dans la fine botte (mines) ou la grande botte (ponts) ne sont pas mélangés avec les simples élèves externes. En revanche, par certains traits, les grandes écoles se montrent semblables à la familia romaine: ceux qui n'appartiennent pas à la communauté par le concours mais évoluent en son sein du fait de leur fonction sont présents sur les monuments. Ils ont, comme il se doit, leur place particulière. (fig. $\mathbf{n}^{\circ} \mathbf{1 2}$ ) Sur l'obélisque de l'École des ponts et chaussées, un emplacement est réservé au personnel administratif. Le monument aux morts des normaliens tombés lors de la seconde guerre mondiale porte le nom d'un agent de service, mais ce dernier est placé en bas de la liste et sa qualité est signalée en italique. Les hiérarchies sont ainsi respectées et la diversité des origines est maintenue. 
Figure 12

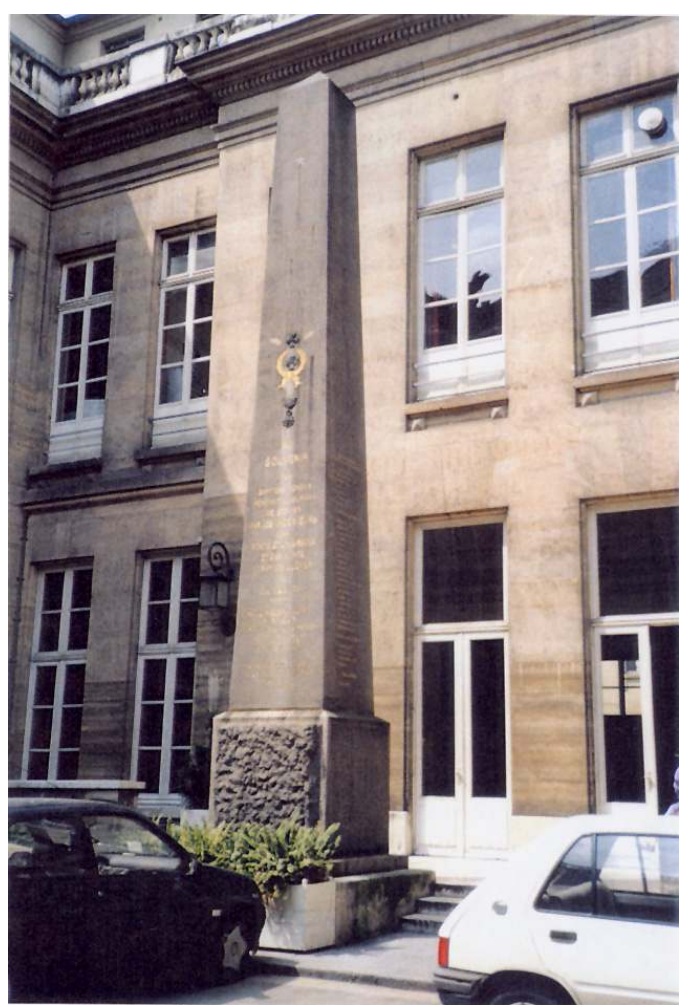

MONUMENT AUX MORTS De L'ÉCOLE NATIONALE DES PONTS ET CHAUSSÉES (PARIS), 1878.

PHOT. CHRISTIAN HOTTIN, 1995.

Si ces inscriptions nous révèlent quelques-uns des traits distinctifs des institutions, c'est à travers les sculptures que se manifeste avec le plus d'éclat l'image que la communauté souhaite donner d'elle-même, c'est là qu'apparaît le mieux, sous la forme particulière du culte des morts, le culte rendu à l'établissement. Les monuments aux morts de la Faculté de médecine sont au nombre de deux. (fig. $\left.\mathbf{n}^{\circ}{ }^{13}\right)$ (fig. $\mathbf{n}^{\circ} \mathbf{1 4}^{2}$ ) (fig. $\mathbf{n}^{\circ}{ }^{15}$ ) Le plus important, œuvre de Bénard, est placé au cœur de l'ancienne École de chirurgie édifiée par Gondoin et montre un jeune médecin des armées assis à l'entrée d'un poste de secours ${ }^{43}$. 
Figure 13

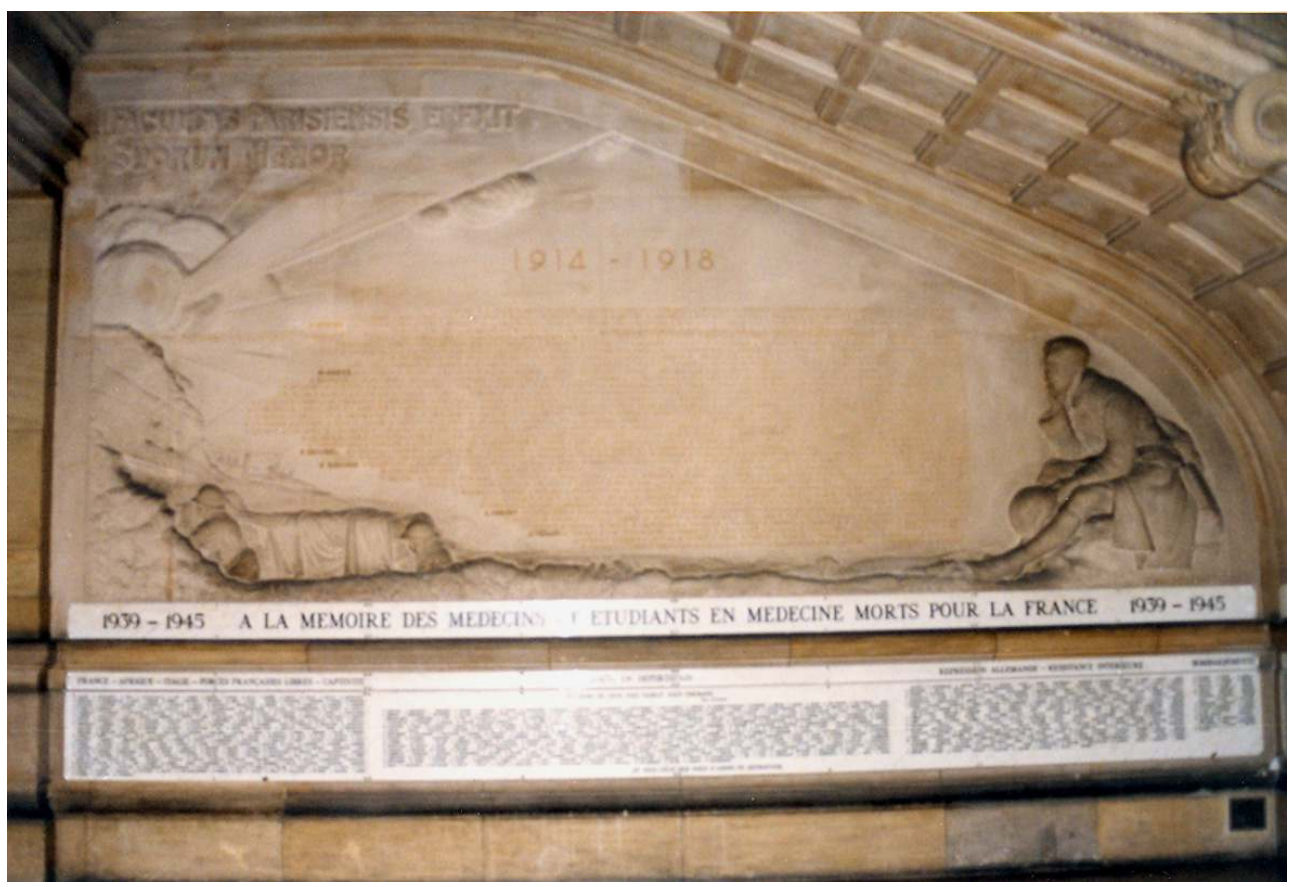

R. BÉNARd, MONUMENT AUX MORTS de LA FACULTÉ de MÉdeCINE (PARIS), 1925. PHOT. PHILIPPE REIX, 1995.

Figure 14

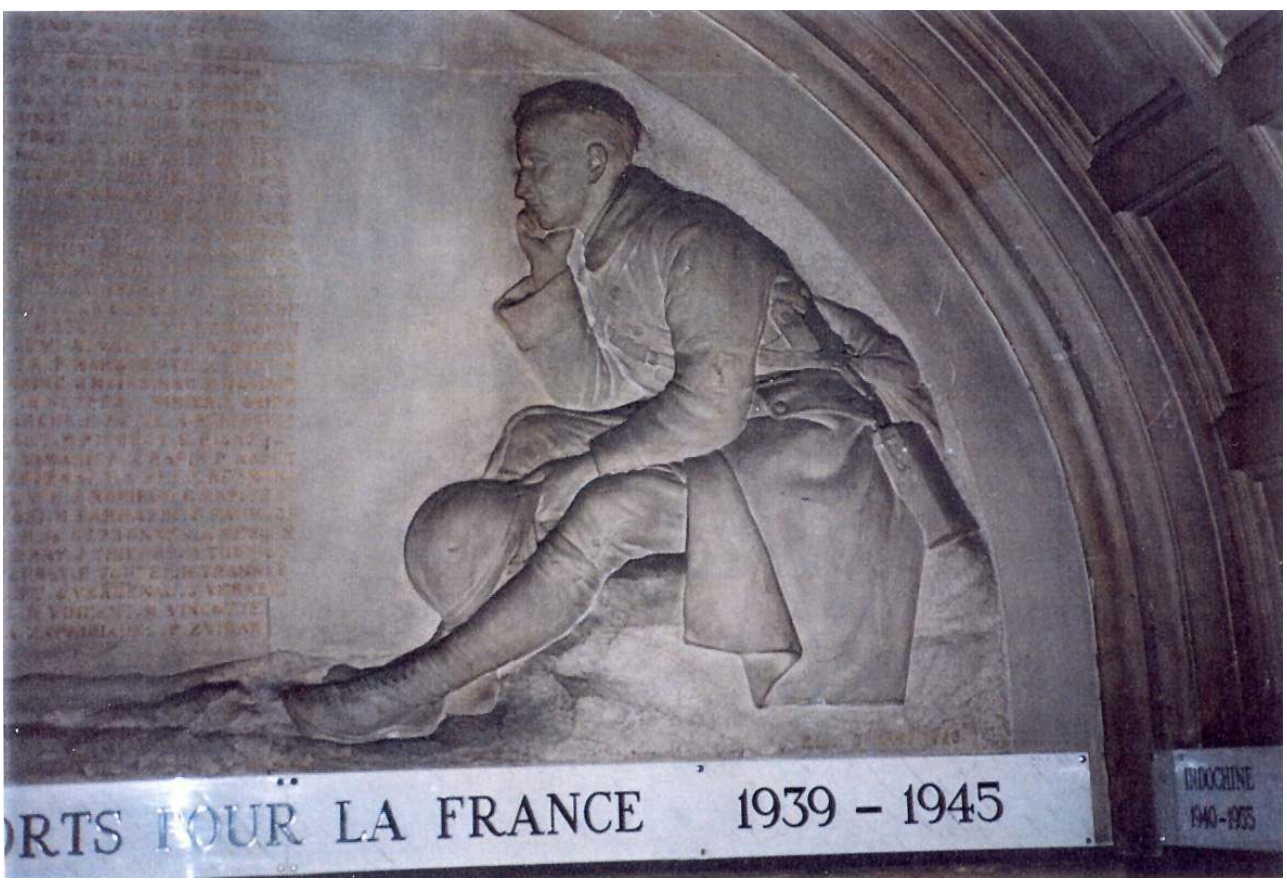

R. BÉNARd, MONUMENT AUX MORTS de LA FACULTÉ de MÉdeCine (PARIS), DÉtAIL, 1925. PHOT. PHILIPPE REIX, 1995. 
Figure 15

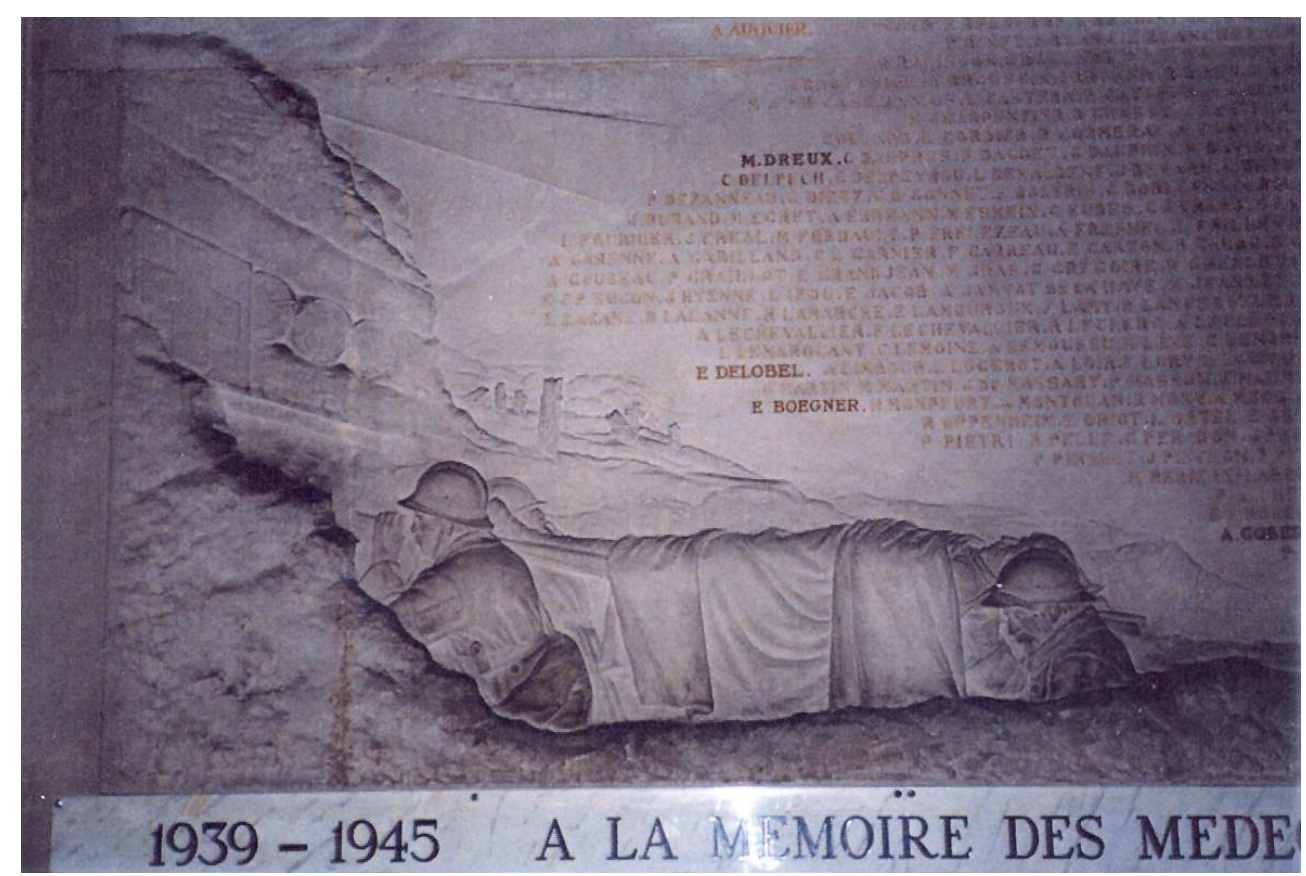

R. BÉNARd, MONUMENT AUX MORTS DE LA FACULTÉ dE MÉdeCINE (PARIS), DÉTAIL, 1925.

PHOT. PHILIPPE REIX, 1995.

L'autre, plus modeste, (fig. $\mathbf{n}^{\circ} \mathbf{1 6}$ ) est un bas-relief du sculpteur Blanchot fixé au mur de façade de l'école pratique. On y voit un médecin secourant un blessé sur le champs de bataille. Dans les deux cas sont sobrement évoquées les conditions de travail difficiles, le danger couru par les médecins, mais aussi leur courage et leur sens du devoir : quelles que soient les circonstances, ils connaissent leur mission et soignent sans relâche, montrant de ce fait les mêmes vertus en temps de paix et en temps de guerre. Cette équivalence entre les qualités civiles et les qualités militaires est véritablement la clef de voûte des représentations universitaires liées à la Grande Guerre. Cet événement formidable a rendu plus visibles les traits distinctifs, acquis (ou même supposés innés) des membres des institutions universitaires ; ces caractères existaient au temps de la paix, ils deviennent plus saillants grâce aux hostilités, qui permettent en outre leur mise en scène dramatique. Il a souvent été fait référence aux monuments des ulmiens et des centraliens dans l'étude du financement et de la construction : l'importance de la souscription et la participation d'anciens élèves à la conception architecturale de l'œuvre témoignent de la charge affective considérable que porte le monument. Ces deux exemples sont également ceux qui illustrent le mieux la participation des monuments aux morts au discours qu'une institution universitaire produit sur elle-même. Le monument n'est plus seulement illustration des vertus militaires et reflet des qualités civiles, mais il se développe un rapport interactif entre espaces civils et militaires, qui sont deux domaines d'expression des caractères particuliers des centraux et des normaliens. Le jeune homme mortellement blessé de Paul Landowski n'est pas une simple allégorie de la jeunesse : le flambeau du savoir qu'il tend en un ultime effort l'assimile explicitement à un intellectuel. Le discours de Lanson rend l'image plus claire encore :

«De la main du camarade défaillant, ils [les jeunes normaliens] prendront le flambeau de l'énergie spirituelle et de la vertu scientifique, et ils le transmettront à la génération future ${ }^{44}$. » 
Figure 16

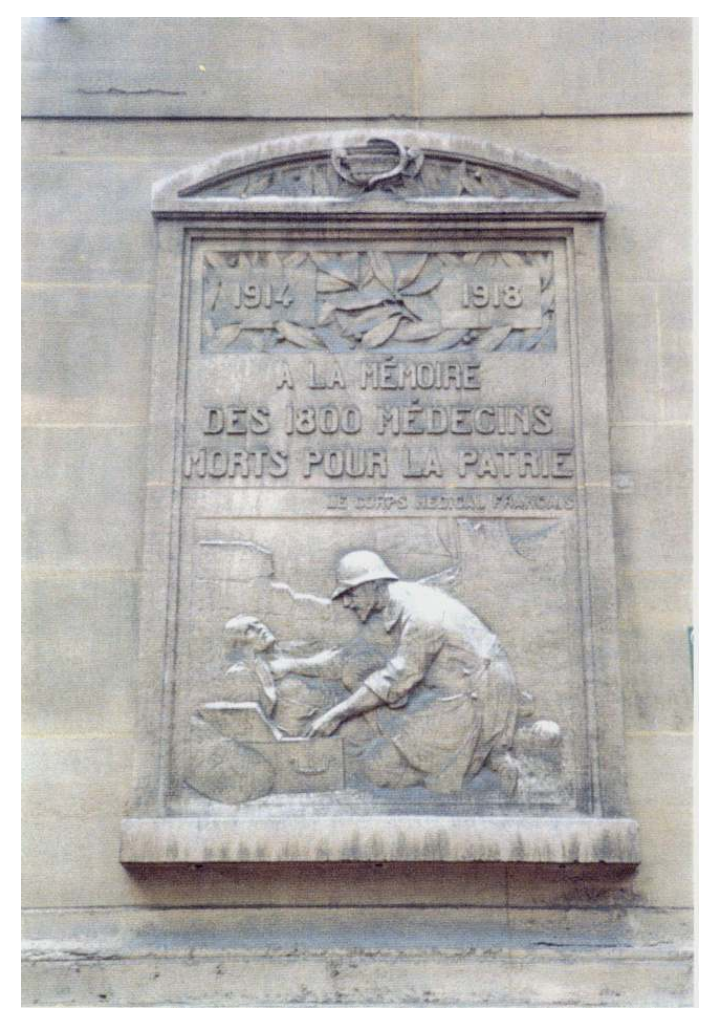

L.L. BLANCHOT, MONUMENT AUX MORTS DES MÉDECINS FRANÇAIS (PARIS, FACULTÉ DE MÉDECINE DE LA RUE DE L'ÉCOLE DE MÉDECINE), S.D.

PHOT. CHRISTIAN HOTTIN, 1995.

Cette personnification est complétée par une série de bas-reliefs montrant les activités des normaliens pendant le conflit. Ils combattent dans l'artillerie, l'aviation et, principalement, l'infanterie. Ce dernier point correspond à la réalité historique, puisque les normaliens furent souvent officiers subalternes d'infanterie, ce qui explique en partie l'importance des pertes subies. Le directeur de l'école n'hésite pas à détailler ces scènes qui doivent rappeler aux anciens les années passées au combat : (fig. $\left.\mathbf{n}^{\circ} \mathbf{1 7}\right)$

«Regardez les bas-reliefs dont l'artiste a encadré la liste de nos morts. Voyez-les : artilleurs à leurs pièces, génie creusant les mines, mitrailleurs ajustant leur engin, infanterie prête à l'attaque, dont le chef a l'œil fixé sur la montre attachée à son poignet, dans l'attente de l'heure " $\mathrm{H}$ " ${ }^{\prime 4}$." 


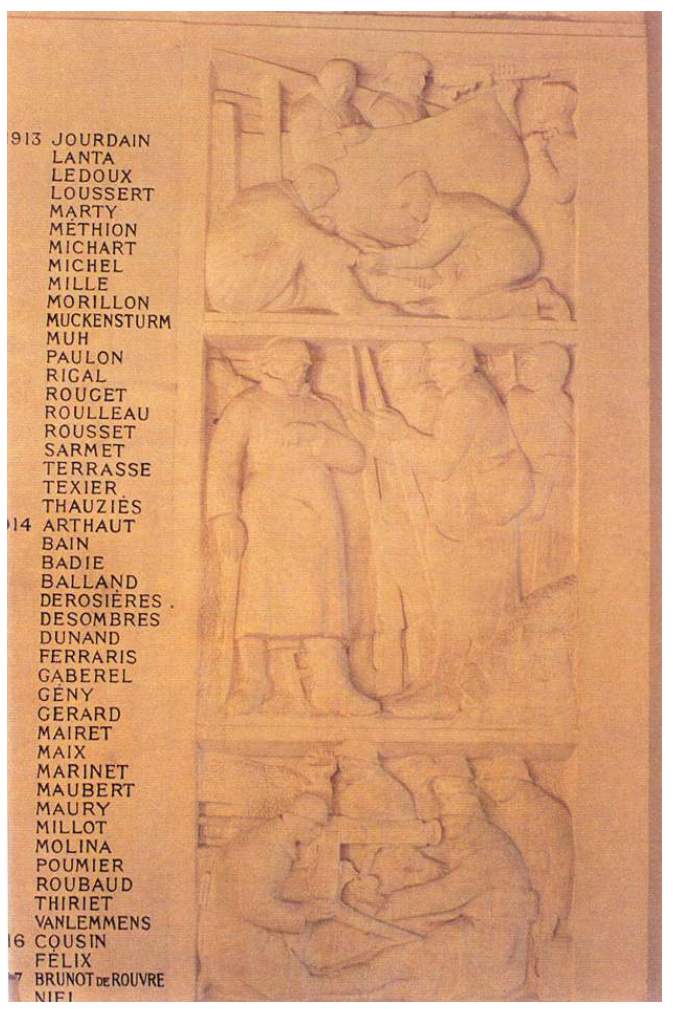

P. LANDOWSKI, MONUMENT AUX MORTS DE L'ÉCOLE NORMALE SUPÉRIEURE (PARIS), DÉTAIL, 1921. PHOT. PHILIPPE REIX, 1995.

Or, ces sculptures n'illustrent pas seulement la bravoure des normaliens en temps de guerre ; le spectacle de leurs activités renvoie au souvenir de leurs occupations civiles, et les mêmes qualités s'observent dans les deux cas :

"[Le monument donne] enfin une leçon de travail méthodique et suivi : rien ne s'achève, rien ne dure que par là (...) Quelle application, quelle gravité dans toutes ces opérations dont le produit sera la victoire ! Rien de tapageur, rien de théâtral : chacun s'oublie dans sa besogne. Ils sont tels que nous les avions connus à la bibliothèque et dans les laboratoires ${ }^{46}$. "

L'œuvre de Paul Landowski ne nous donne pas, dans son état définitif, l'illustration de ce parallèle rhétorique. Mais un projet de l'artiste laisse penser que cette solution fut un temps envisagée, puisqu'un modèle en plâtre montre dans les bas-reliefs de la partie supérieure du monument des jeunes gens au travail, studieusement penchés sur leurs livres ou leurs paillasses de laboratoire ${ }^{47}$. La scène retenue pour figurer sur l'œuvre définitive est en fait encore plus audacieuse et chargée de sens, puisqu'elle place les normaliens morts dans les Champs-Élysées des anciens, s'entretenant avec de graves philosophes, tandis que leurs cadets gravent dans la pierre des paroles honorant leur mémoire ${ }^{48}$. Ainsi, le normalien manifeste une attitude spécifique dans la vie comme dans la mort, il se conduit comme tel du berceau jusqu'à la tombe et son sérieux, son intelligence ou sa persévérance sont les signes patents de l'appartenance à une élite qui n'est ni intellectuelle ni sociale mais tout simplement humaine. Les thèmes traités dans le monument aux morts rappellent les paroles prêtées au président Georges Pompidou, qui disait que l'on ne devenait pas normalien, mais que l'on naissait normalien, comme on naissait prince du sang. Cette œuvre est à mettre en relation avec une autre, d'autant plus 
intéressante et originale qu'elle fut exécutée par des élèves de l'école : la fresque de la turne François-Poncet ${ }^{49}$. Cette frise, conçue et exécutée pendant la préparation de l'agrégation, met en scène les normaliens et les sorbonnards. Tous se préparent au concours, mais les normaliens seuls font preuve d'une discipline et d'une ardeur qui débouchent sur le succès. Ils se rendent à l'oral en défilant, alignés comme à la parade, rangés par discipline. Les qualités du normalien inscrites dans les catégories du discours officiel trouvent donc un écho, sur le mode humoristique de la caricature, dans le discours des étudiants : à tous les niveaux, la spécificité de l'institution est affirmée et déclinée selon des critères identiques. L'École centrale des arts et manufactures offre un autre exemple d'institution dont le monument aux morts, pièce centrale du système de représentation de la communauté, exalte les qualités de l'école tout en rendant hommage aux défunts. Les soldats figurés par Marcel Gaumont sur les côtés de l'arc de triomphe servent dans les armes qui accueillirent les centraliens au cours de la Grande Guerre, l'artillerie et l'aviation. Ils sont représentés devant un avion et un canon. En effet, le rôle des centraux au sein de l'artillerie est mis en relief dans de nombreux textes, à commencer par la citation à l'ordre de l'armée qui couronne la porte d'entrée : (fig. $\mathbf{n}^{\circ} \mathbf{1 8}$ )

«L'École centrale des arts et manufactures, par son organisation militaire originale, a constitué une véritable pépinière d'officiers d'artillerie de complément dont la science et l'héroïsme ont, au cours de la guerre, grandement et glorieusement contribué à assurer la victoire ${ }^{50}$. »

Figure 18

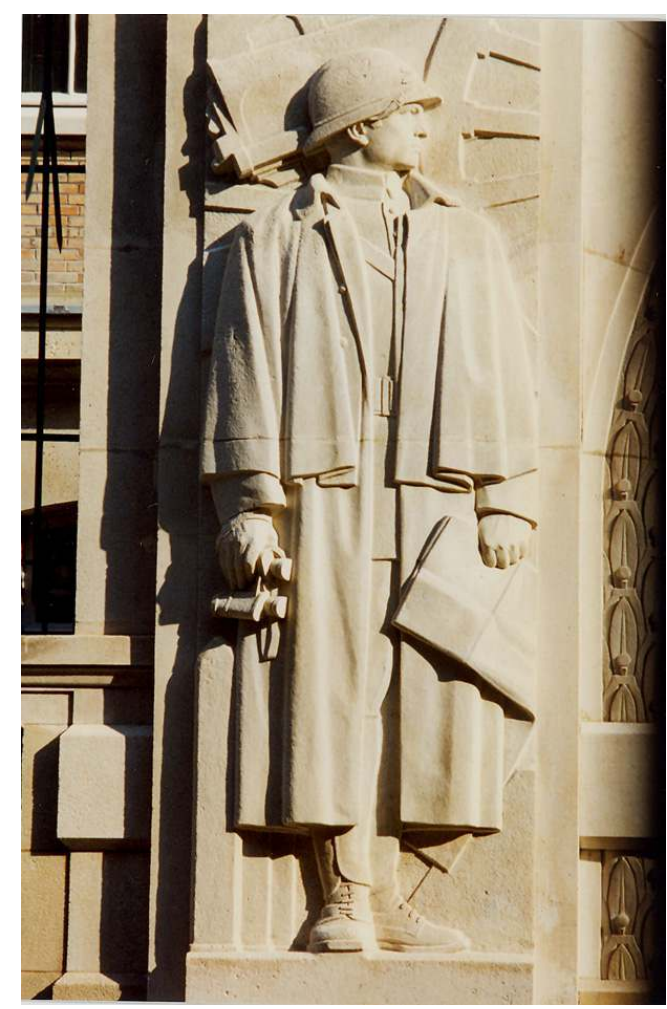

M. GAUMONT, MONUMENT AUX MORTS DE L'ÉCOLE CENTRALE Des ARTS ET MANUFACTURES (PARIS), DÉTAIL, 1923.

PHOT. PIERRE HAgARD, 1996.

Nous retrouverons ici le balancement rhétorique traditionnel entre science et courage. La première fait partie intégrante de l'univers des universités et des grandes écoles, tandis 
que le second sert, dans tous les discours, à caractériser le soldat français, quelle que soit son origine. Qu'en est-il de la bravoure propre aux centraliens et des services particuliers rendus par l'école ? Ce thème est amplifié par d'autres moyens. Léon Guillet, dont le directorat est perçu dans l'histoire de l'école comme un véritable règne, au cœur d'une ère particulièrement faste pour l'institution, créa un musée des centraux destiné à glorifier l'institution à travers l'évocation de ses grandes heures et de ses membres les plus prestigieux. Une salle était entièrement réservée au souvenir de la première guerre mondiale, et la phrase suivante était peinte sur le mur, en gros caractères :

«Et alors que cette multiplication infinie des régiments et de toutes les formations entraînait dans l'artillerie une pénurie effroyable d'officiers, on peut dire, je le répète, que l'École centrale a sauvé l'artillerie française ${ }^{51}$. »

Mais c'est un autre thème de l'œuvre de Marcel Gaumont qui permet d'avoir une juste idée du système de représentation de l'École centrale: celui de la machine. Plus précisément, le centralien est mis en relation avec la machine qu'il crée et qu'il maîtrise. C'est le cas dans le monde militaire, puisque l'artilleur centralien manie son canon de 75 avec précision et efficacité. Mais il est aussi l'ingénieur qui possède une connaissance intime de la machine, qui est capable non seulement de la réparer mais encore de la créer. Celle-ci, en tant que symbole universel de la modernité, transmet à l'ingénieur sa caractéristique principale. De même que ses qualités intellectuelles conféraient au normalien l'intemporalité et l'éternité, le centralien sera moderne. À travers son rapport à la machine s'exprime sa permanente et parfaite adaptation aux circonstances sans cesse évolutives du monde contemporain. Le thème sera traité à plusieurs reprises à l'École centrale des arts et manufactures. Les fresques peintes par Leprince-Ringuet sur les murs du réfectoire introduisent un balancement chronologique qui se retrouve dans d'autres œuvres ${ }^{52}$. Chaque scène figure un dieu de l'olympe muni de ses attributs et occupé à domestiquer ou à mettre en valeur la nature. Démeter cultive les céréales, Zeus tient la foudre, etc. Sous ces grandes figures sont peints des machines modernes (tracteurs ...) ou des ingénieurs (ceux qui accompagnent Zeus s'activent devant des turbines). La mise en perspective chronologique et la comparaison mythologique flatteuse donnent au thème de la modernité plus de force que dans le monument aux morts. Ce parti est également exploité sur les couvertures des programmes du centenaire de l'école, en 1929. Les dessins de Paul Furiet et de Pierre Leprince-Ringuet opposent le centralien de 1829 et celui de 1929, chacun étant accompagné des machines de son époque, qu'il s'agisse du steamer ou du paquebot transatlantique... La notice de la séance solennelle du 26 mai 1929, une autre œuvre de Pierre Leprince-Ringuet, s'en tient en revanche à l'illustration de la modernité : un graphisme sobre, des formes géométriques inspirées des arts décoratifs, une accumulation de symboles du monde contemporain, la ruche, autre élément essentiel et polysémique de la représentation de l'École centrale, et, au cœur de ce foisonnement ... le monument aux morts ! Cet arc de triomphe trouve ici l'aboutissement de son exploitation comme élément du système de représentation : il n'est plus un moyen parmi d'autres de représenter l'école, mais le symbole de celle-ci. La gloire des morts et celle de l'école ne font plus qu'une, et le monument, habilement stylisé, cesse de donner une image de l'école pour devenir l'école. À Centrale comme à Normale le discours sur l'institution finit par l'emporter sur l'évocation de la Nation, le culte des morts s'affirme comme un culte particulier. 


\section{L'usage des monuments et l'entretien du culte} monument. Cette cérémonie marque au contraire le début d'une nouvelle phase des rapports entre la communauté et ses défunts, puisqu'elle bénéficie désormais, grâce à l'œuvre d'art, d'un point d'ancrage pour les célébrations périodiques et l'entretien quotidien de la mémoire. Le monument s'inscrit dans le paysage de l'école, il tient sa place aux côtés des autres œuvres d'art, peintures des amphithéâtres et des espaces de réception, bustes qui ponctuent le discours architectural (vestibules, salles des actes, façades...), monuments qui garnissent les cours. Or, la place assignée aux monuments aux morts n'est pas choisie uniquement en fonction de critères architecturaux, mais également d'après des choix affectifs. Ceux-ci ne sont pas systématiquement installés dans les espaces de représentation les plus ouverts sur le monde extérieur, tels que les vestibules d'entrée. Ils ne prennent pas non plus place dans ces lieux du pouvoir de la communauté que sont les salons et les bureaux de l'administration ou les salles des autorités. En revanche, ils sont fréquents dans les lieux quotidiennement utilisés par les membres du groupe, élèves et enseignants. Les morts ont leur place au milieu des vivants, au sein de leur famille et, ce qui parait très important, en un endroit où ils vécurent les années heureuses de leur scolarité :

« Nous avons élevé ce monument à la mémoire et à la gloire de nos camarades dans ce jardin où s'est écoulée la plus belle partie de leur jeunesse ${ }^{53}$. "

Plus que le rapport au monde extérieur, c'est la relation particulière entre les jeunes générations et les aînés décédés qui est privilégiée à travers le choix du site : le vestibule de l'aile de la bibliothèque à l'École normale, le jardin central à l'Institut agronomique (où les bustes et statues des plus grandes figures de l'établissement sont installés tout autour du bas-relief, comme pour participer à l'hommage du groupe à lui-même), le jardin botanique à l'École supérieure de pharmacie. Le monument des centraliens, quant à lui, est tourné vers l'extérieur, puisqu'il encadre l'entrée des élèves, située rue Conté ; (fig. $\mathbf{n}^{\circ}$ 19) mais il s'impose encore plus aux étudiants qui, chaque matin, pénètrent dans l'école en passant sous la Victoire casquée et ailée qui habite le tympan et en longeant la liste des disparus. En un lieu beaucoup plus intime, la grande salle de cours, nous découvrons le monument aux morts de l'École des chartes. Ce n'est pas en raison de l'exiguïté des locaux qu'il est fixé sur le mur, au-dessus de la chaire : il se trouve ainsi au cœur de l'institution, les morts restent présents sur le lieu de leurs études et dans la pièce où ils soutinrent leurs thèses. Les discours officiels soulignent une autre valeur symbolique de cet emplacement :

«Le monument que nous avons voulu placer ici, dans la salle principale des cours, centre véritable de la vie de l'école, et où se tiennent, chaque année les soutenances des thèses, rappellera à toutes les générations qui se succéderont dans cette enceinte l'héroïque sacrifice de ceux des nôtres qui sont tombés pour le salut de la patrie et pour la sainte cause du droit. Nul ne franchira le seuil de cette salle sans reposer ses regards sur cette inscription. Elle apparaîtra, sur cette muraille, tout ensemble comme l'affirmation continue de notre reconnaissance et de notre tendresse infinie à l'égard de nos morts, et comme un précepte, un symbole toujours présent du devoir et de l'abnégation patriotique. Auprès de l'antique tableau noir, où nous avons vu, jeunes élèves, tant de démonstrations savantes, se dressera désormais un autre tableau, celui qui dira les gloires de notre école, en 
présentant, par une simple énumération de noms, la plus belle page de son histoire 54. »

Figure 19

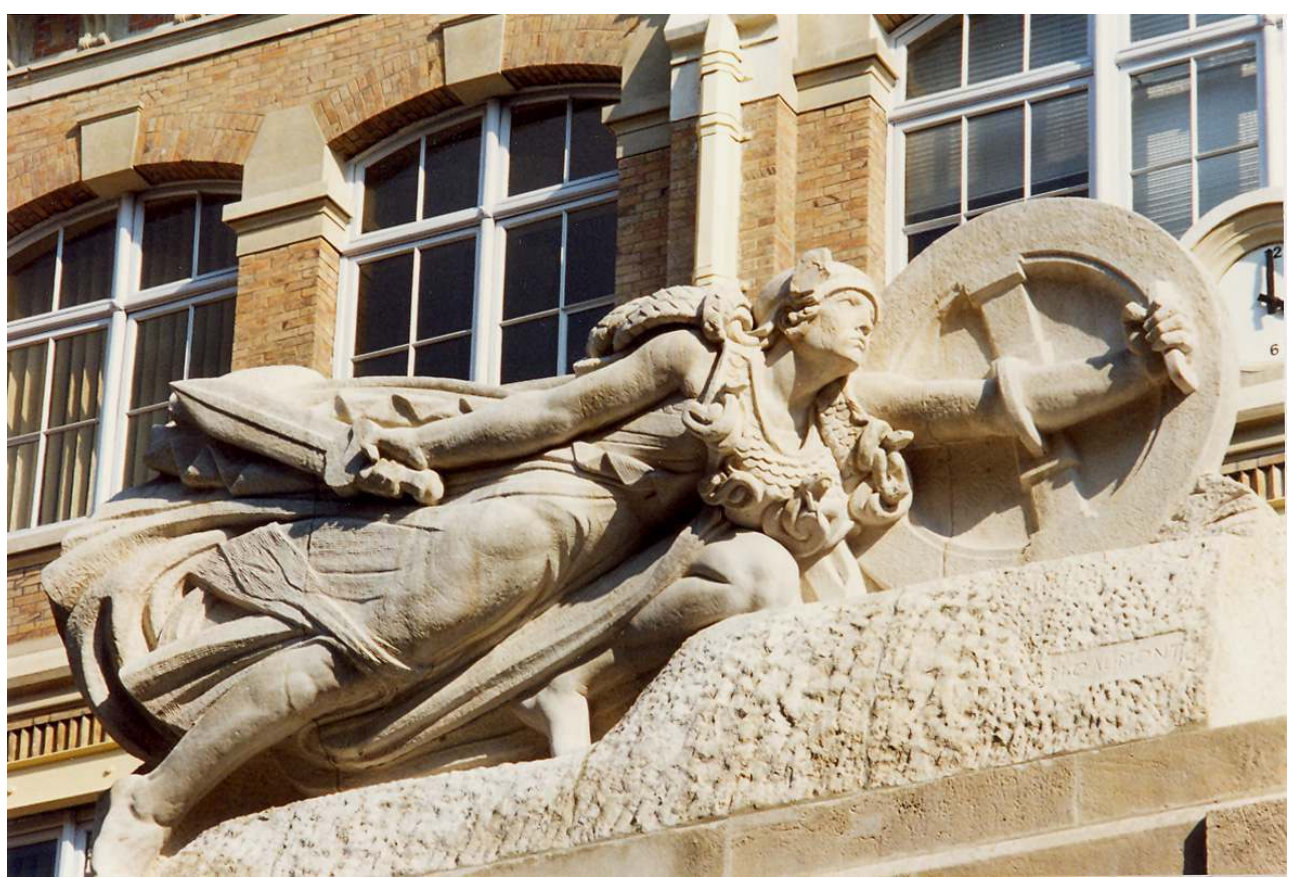

M. GAUMONT, MONUMENT AUX MORTS DE L'ÉCOLE CENTRALE DES ARTS ET MANUFACTURES (PARIS), DÉTAIL, 1923.

PHOT. PIERRE HAgARD, 1996.

l'exemplarité de leur sacrifice. Nous retrouvons au passage le balancement entre le discours sur l'école et le discours sur la Nation, mais la première occupe une place bien plus considérable. Certes, la gloire de l'école se situe dans l'accomplissement du devoir patriotique, mais elle l'emporte sur le culte de la Nation: à travers le monument aux morts, c'est l'école qui est glorifiée (les savantes démonstrations) et ses lieux qui sont sanctifiés (évocation émue du tableau noir). $\mathrm{du}$ culte des morts sera, par excellence, le lieu du souvenir et de l'exemple. Double mission bien comprise par les jeunes générations, même si, après la seconde guerre mondiale, celles-ci reconnaissent qu'elles n'ont pas toujours suffisamment tenu compte de la leçon donné par les anciens :

« Il faut l'avouer, nous avions peut-être trop pris l'habitude de voir sans la regarder cette plaque qui témoigne depuis plus de vingt-cinq ans du sacrifice de nos anciens. Pourtant il n'en est pas un de nous, j'en suis sûr, qui, un jour ou l'autre, n'ait arrêté sa pensée, ne fut-ce qu'un instant, devant cette liste glorieuse : nous nous étions dits, alors, combien ces morts étaient belles et que ces hommes, sans doute, avaient eu la consolation en tombant dans la bataille, d'espérer au moins que leur sacrifice n'était pas inutile ${ }^{55}$."

On pourrait ainsi multiplier les références à des textes insistant sur la valeur exemplaire du sacrifice consenti et sur l'importance du monument dans l'entretien de cette mémoire : 
« Nous avons voulu qu'il [le monument] fût placé dans l'école même afin que les générations d'élèves qui passeront devant lui aient toujours présent à l'esprit le sacrifice de leurs aînés. Si la France était de nouveau en danger, il dicterait leur devoir aux jeunes en rappelant comment leurs aînés ont compris et accompli le leur ${ }^{56}$. »

Cependant, le monument ne témoigne pas seulement pour les membres de la communauté, il parle également au monde extérieur, à la Nation. Et le message qu'il délivre alors n'est pas uniquement celui de la fusion avec le reste du pays par le sang versé, il est également un plaidoyer en faveur de l'école, une défense de cette dernière face à ses ennemis. Le culte rendu aux morts ne se limite donc pas à l'action des vivants en faveur des disparus, puisque ces derniers peuvent encore agir pour leurs successeurs et les défendre. Les morts fondent la gloire de l'école, ils désarment par avance toutes les critiques:

«On a pu déplorer naguère l'individualisme, l'esprit critique des normaliens: ils répugnaient à toute discipline, comme si leur personnalité trop forte eût supporté impatiemment les contraintes de l'esprit de corps. La guerre nous a rapprochés; elle a ranimé la vie morale de l'école : celle-ci sort plus forte de l'épreuve. Qui donc oserait aujourd'hui proposer de supprimer une institution qui à son prestige d'autrefois vient d'ajouter cette douloureuse gloire ? Cette gloire, nous la devons à nos morts ${ }^{57}$."

Le président de l'association des anciens élèves de l'École normale supérieure de SaintCloud utilise également cette idée, en mettant en outre en avant l'importance des morts dans la consolidation d'une institution encore fort jeune, comme si le sang répandu faisait accéder l'institution à l'âge adulte :

«Leurs rangs ont été plus que décimés par la guerre (...) Ainsi la plus petite et la plus modeste sans doute des grandes écoles se place au premier rang par le tribut qu'elle a payé à la victoire du droit (...) Les noms de ces héros gravés sur nos murs veilleront aussi sur notre école. Si jamais elle était menacée, elle n'aurait qu’à montrer ces listes funèbres. Le foyer où tant d'éducateurs ont puisé la flamme qui les a consumés ne sera pas dispersé ${ }^{58}$.»

Les fonctions du monument, suggérées par son emplacement dans la géographie de l'institution et explicitées par les discours d'inauguration, sont donc multiples : il associe culte de la Nation et culte de l'école, tout en privilégiant volontiers ce second aspect, il est le lieu du souvenir et de l'exemple, et les morts qu'il honore sont une protection pour le reste de la communauté, un moyen d'asseoir sa place dans la société. Mais le monument n'est qu'une forme parmi d'autres du culte des morts. Nous avons vu qu'il était comme le rappel permanent du devoir accompli et de la mort consentie et que, par sa présence, il diffusait le souvenir des défunts dans les lieux qu'il occupait. Cela ne suffit cependant pas: l'œuvre d'art, même si elle porte sur des plaques voisines les noms de tous les disparus, ne restitue que très imparfaitement l'individualité de chaque individu. Quels étaient leurs visages? Quelles furent leurs vies? Pour rendre compte de la diversité des existences, le monument doit se faire personnel et portatif : c'est le livre d'or.

La publication d'un livre d'or de l'école n'a jamais été perçue comme une nécessité, à la différence de l'érection du monument aux morts. Seules quelques institutions, telles que l'École centrale, l'Institut agronomique, l'École des chartes ou l'École normale supérieure d'enseignement primaire de Saint-Cloud se sont lancées dans cette entreprise. Bien souvent, le livre d'or prend appui sur le monument aux morts : à l'École des chartes et chez les normaliens de Saint-Cloud, il s'ouvre par le récit de l'inauguration, auquel sont joints les textes des différents discours prononcés à cette occasion. Mais le livre prolonge 
le monument, il l'explicite en livrant, pour chaque défunt, une notice nécrologique, parfois assortie d'une photographie (cette particularité est même précisée en titre dans le cas du livre d'or de l'École des chartes). Chaque famille peut donc retrouver son mort, chaque camarade son compagnon de promotion. Le livre d'or de Saint-Cloud donne en annexe des cartes indiquant, à proximité des champs de bataille, les sépultures des anciens élèves dont les corps n'ont pas été enterrés auprès de leurs proches. Un pèlerinage devient ainsi possible pour les amis et les familles, pèlerinage dans lequel l'école trouve naturellement sa place, puisqu'elle constitue une sorte de relais entre la cellule familiale et le monde extérieur, comme il est suggéré dans une préface :

«Les parents de nos enfants morts ont un sentiment profond de solidarité dans la douleur qui unit à eux cette maison, ils considèrent l'école comme une autre famille qui avec eux et après eux doit se souvenir, honorer leurs fils, les donner en exemple. Quand ils ont pu entreprendre le voyage vers la sépulture de leur enfant, Saint-Cloud a été une étape de réconfort dans leur douloureux pèlerinage ${ }^{59}$."

Avec le livre d'or la famille du défunt apparaît. De même que le discours des monuments plaçait le mort entre l'école et la Nation, celui des livres d'or le met entre ses proches et son école. Dans cet extrait, l'individu honoré semble tiré tantôt vers son institution, tantôt vers les siens (nos enfants et leur enfant). L'établissement se présente comme une autre famille, capable d'englober la première, biologique, mais possédant en outre un atout considérable : étant un corps sans cesse renouvelé, il survivra aux parents naturels et, après leur mort, gardera seul le souvenir du disparu au combat. Néanmoins, les notices nécrologiques n'ont pas pour unique but de juxtaposer les biographies. Tous ces textes, que les auteurs en aient manifesté ou non l'intention, insistent sur certaines qualités des individus, souvent les mêmes, pour constituer in fine, une fois la lecture achevée, un grand portrait en mosaïque de la personnalité de l'institution. À travers la singularité des parcours, ce sont encore les images de l'école que l'on découvre, l'accumulation analytique des textes venant remplacer la vision synthétique offerte par les sculptures. Maurice Donnay, évoquant le livre d'or de l'École centrale des arts et manufactures, a mis en relief l'impression d'unité qui se dégage de l'ensemble des notices :

«[Ce livre] Laurier magnifique, dont toutes les fleurs sont semblables, sans qu'aucune soit tout à fait pareille, livre d'or empourpré de taches de sang, égalité dans l'exceptionnel, nivellement par les plus hautes vertus ${ }^{60}$.»

De ce fait, les livres d'or offrent une nouvelle approche des systèmes de représentation des institutions. Où le discours en images n'existe pas, le discours écrit compense cette absence; parfois, les textes viennent affiner ce qui n'était que suggéré dans les sculptures. On retrouve le principe de la conformité entre la formation professionnelle et les qualités manifestées au combat, toutes donnant à penser que le comportement de l'individu est le reflet de la communauté qui était la sienne :

«C'est avec passion que tous ces agros se sont élancés à la défense de ce sol qu'ils avaient d'autant plus de raison d'aimer qu'ils le connaissaient jusque dans ses éléments constitutifs et qu'ils l'avaient analysé, fouillé, retourné et enrichi par leur travail quotidien depuis leur sortie de notre chère école ${ }^{61}$. "

Quelle que soit la forme prise par le culte des morts, dans le marbre ou sous la plume des membres des institutions, l'hommage à la Nation défendue par ses fils et l'affirmation de la spécificité du groupe demeurent deux thèmes étroitement entrelacés. Ce dialogue se poursuivra jusqu'à la seconde guerre mondiale. Celle-ci marquera une rupture très nette, tant sur le plan esthétique que sur le plan stylistique. 
41 Dans les établissements d'enseignement supérieur, le culte des morts de la Grande Guerre n'est plus aujourd'hui qu'une survivance, à l'exception du cas particulier des écoles militaires. En revanche, quelques soixante-dix ans après leur installation, les monuments subsistent presque tous. Après n'avoir suscité aucun intérêt pendant des décennies, après avoir connu de nombreux déboires (les plaques de l'ancienne École coloniale furent déposées lors de la disparition de l'établissement, le monument aux morts des normaliens fut mutilé en mars 1971 lors d'une fête étudiante), ils sont aujourd'hui restaurés : le monument aux morts des centraux a été nettoyé par le Conservatoire national des arts et métiers, nouvel occupant des bâtiments de la rue Montgolfier, et la sculpture de Paul Landowski a retrouvé en 1996 le bras qui lui manquait depuis vingt-cinq ans. Toujours présentes physiquement, ces œuvres ont en revanche perdu une bonne part de leur signification, puisque, si le discours concernant la Nation reste largement compréhensible, celui attaché aux institutions n'est plus guère perceptible, tant se sont modifiés les systèmes de représentation des établissements d'enseignement supérieur ${ }^{62}{ }^{63}$.

\section{NOTES}

1. - Leur sang précieux resplendit dans la gloire. Inscription figurant sur le monument aux morts de l'École nationale des chartes.

2. - ROMAINS, Jules. Vorge contre Quinette. Paris, 1958 (Les Hommes de bonne volonté, vol. III, t. 17), p. 478.

3. - ROMAINS, Jules. Vorge contre Quinette. Paris, 1958 (Les Hommes de bonne volonté, vol. III, t. 17), p. 479 et 480 .

4. - Entre plusieurs études consacrées à ces œuvres, citons : BUSSCHER, Jean-Marc de. Les Folies de l'industrie. Bruxelles, 1981. Le contenu en est très personnel, mais les photographies sont nombreuses et souvent de grande qualité. Voir aussi : ROZE, Anne. Les Lieux de la Grande Guerre. Paris, 1996.

5. - La contribution d'Antoine Prost à l'entreprise de Pierre Nora est la meilleure synthèse sur le sujet : PROST, Antoine. « Les monuments aux morts ». Dans Les Lieux de mémoire (Pierre Nora dir.), t. 1 La République. Paris, 1984, p. 195-225. Voir aussi : BECKER, Annette. Les monuments aux morts. Patrimoine mémoire de la Grande Guerre. Paris, 1988.

6. - Le monument aux morts de l'église Saint-Thomas d'Aquin constitue un exemple intéressant : y apparaissent toutes les familles aristocratiques du Faubourg Saint-Germain.

7. - Citons à titre d'illustration celui du quartier Saint-Aubin à Toulouse : bien que placé devant l'église du même nom, il est manifestement d'inspiration républicaine et patriotique, comme en témoigne le coq gaulois perché sur la stèle.

8. - Il n'est que de voir ceux des cheminots, placés, il est vrai, après 1945 , dans la plupart des gares de France pour se convaincre de l'importance que peut prendre ce phénomène.

9. - On peut songer au rôle alors rempli par Paul Dupuy, secrétaire de l'École normale supérieure, qui entretint une abondante correspondance avec les anciens élèves mobilisés, renseignant chacun sur la situation des autres et incarnant ainsi pratiquement dans sa personne ce lien.

10. - Association Amicale de l'Institut National Agronomique, compte-rendu des cérémonies commémoratives en mémoire des anciens élèves et élèves de l'Institut National Agronomique. Paris, [1917]. 11. - GUÉRIN, M. « Ceux de 19-20 ». Dans Rue d'Ulm (Alain Peyrefitte, dir.), 1994. 
12. - Discours du Président de la République à la séance de rentrée de l'École normale supérieure (23 mars 1919). Paris, 1919. Et Discours du Président de la République à l'École Polytechnique lors de la réception des élèves de retour du front (4 mai 1919). Paris, 1919.

13. - Sur l'École normale supérieure et la Grande Guerre, voir: MASSON, Nicole. L'École Normale Supérieure ou les Chemins de la liberté. Paris, 1994 (Coll. Découvertes Gallimard), p. 48 et 49.

14. - ROMAINS, Jules. Vorge contre Quinette. Paris, 1958 (Les Hommes de bonne volonté, vol. III, t. 17), p. 478.

15. - Arch. nat., F 21 4850, Dossier de l'École normale supérieure. Lettre du directeur Ernest Lavisse au directeur des Beaux-Arts, datée du 5 août 1919.

16. - Arch. nat., F 21 4850, Dossier de l'École normale supérieure. Minute de la réponse du directeur des Beaux-Arts à Ernest Lavisse, non datée.

17. - Parmi les œuvres étudiées, il n'existe qu'une exception: le monument aux morts du Conservatoire national de musique et de déclamation. Voir: Arch. nat., F 21 4235, Dossier de commande à Pierre Lenoir du monument aux morts du Conservatoire.

18. - Ce qu'indiquent les souscriptions des monuments aux morts, les souscriptions en vue de la fonte d'un buste ou de l'érection d'une statue le montrent tout aussi bien: on y retrouve l'expression de la cohésion du groupe et de l'étendue de son influence. Voir, par exemple : Arch. nat., F 21 4852, Dossier de l'Institut Agronomique. Souscription en vue de l'érection d'un monument à Eugène Risler; Arch. nat., 61 AJ 63, École normale supérieure, dossier des commémorations. Souscription pour le buste de Fulstel de Coulanges.

19. - Ces chiffres sont cités par Annette Becker pour les monuments de Compiègne (60 000 francs) et de Beauvais (100 000 francs). BECKER, Annette. Les monuments aux morts. Patrimoine mémoire de la Grande Guerre. Paris, 1988, p. 10.

20. - Arch. nat., 61 AJ 63, École normale supérieure, dossier des commémorations. Dossier de la souscription ouverte en vue de l'érection du monument aux morts, liste des souscripteurs.

21. - En 1938, par exemple, la direction de l'École nationale des chartes traite directement avec le marbrier et graveur Émile Pézard en vue de la pose d'une plaque en pierre de Comblanchien, moyennant la somme de 800 francs. Archives de l'École nationale des Chartes, conservées à l'École, carton concernant les bâtiments de l'École.

22. - Arch. nat., 61 AJ 63, École normale supérieure, dossier des commémorations. Souscription ouverte en vue de l'érection du monument aux morts, liste des souscripteurs.

23. - Si elle était disponible, la liste des souscripteurs pour le monument des centraliens révélerait probablement un phénomène semblable, mais d'une tout autre ampleur: on sait en effet que, au sein du comité, se trouvaient des représentants de grandes entreprises dirigées par d'anciens élèves de l'établissement.

24. - Arch. nat, 61 AJ 63, École normale supérieure, dossier des commémorations. Pour ces deux derniers personnages, soulignons que leur action auprès de l'E.N.S. n'est qu'un aspect d'une vaste entreprise de mécénat intellectuel: Albert Kahn a également financé un centre de documentation sociale à l'E.N.S., et on ne compte pas le nombre des fondations de la marquise Arconati-Visconti : Institut de Géographie, prix Auguste-Molinié à l'École des chartes, etc.

25. - L'État ne paraît pas s'être manifesté dans l'affaire du monument aux normaliens. En revanche, il est fait état d'une subvention de 1000 francs, concédée pour la réalisation du monument de l'École des Arts Décoratifs. Arch. nat., F 21 4850, Dossier de l'École des arts décoratifs. Lettre de remerciements du comité chargé de la souscription au directeur des BeauxArts, 30 juin 1928.

26. - Par exemple : École Normale Supérieure de Saint-Cloud, livre du souvenir, 1881-1906. Paris, 1906.

27. - BONNARIC, M. (président de l'association des anciens élèves). «Discours d'inauguration du monument aux morts ». 1914-1918, Livre d'or de l'École Normale Supérieure d'Enseignement Primaire de Saint-Cloud. Paris, 1921, p. X. 
28. - BONNARIC, M. (président de l'association des anciens élèves). « Discours d'inauguration du monument aux morts ». 1914-1918, Livre d'or de l'École Normale Supérieure d'Enseignement Primaire de Saint-Cloud. Paris, 1921, p. XI.

29. - Arch. nat, 61 AJ 63, École normale supérieure, dossier des commémorations.

30. - Par exemple : DONNAY, Maurice. Centrale. Paris, 1927, p. 104.

31. - L'Illustration, $2^{\mathrm{e}}$ semestre 1923, p. 465.

32. - Cette toile est actuellement exposée dans les locaux de la direction de l'École centrale des arts et manufactures, à Châtenay-Malabry.

33. - PROST, Antoine. «Les monuments aux morts ». Dans Les Lieux de mémoire (Pierre Nora dir.), t. I, La République. Paris, 1984, p. 195-225. Quelques monuments de ce type sont représentés page 198. Ils semblent particulièrement représentatifs des monuments patriotiques à la Victoire.

34. - ISAN, Raymond. Paul Landowski. Paris, 1933, p. 10.

35. - POINCARÉ, Raymond. Discours du Président de la République à la séance de rentrée de l'École normale supérieure (23 mars 1919). Paris, 1919, n. p.

36. - LE NORMAND-ROMAIN, Antoinette. Mémoires de marbre, la statuaire funéraire en France (1804-1914), catalogue de l'exposition organisée par la Bibliothèque historique de la ville de Paris, juillet-septembre 1995. Paris, 1995.

37. - PROST, Antoine. «Les monuments aux morts ». Dans Les Lieux de mémoire (Pierre Nora dir.), t. I, La République. Paris, 1984, p. 195-225, p. 217.

38. - « Monument aux morts de la Faculté de Pharmacie ». L'Illustration, $\mathrm{I}^{\mathrm{er}}$ semestre 1926, p. 263.

39. - GAZIOT, P. «Discours d'inauguration du monument aux morts de l'Institut national agronomique » Dans 1914 -1918, À la mémoire des anciens élèves de l'Institut national agronomique morts pour la défense du sol français. Paris, 1921, p. 21.

40. - Antoine Prost et Annette Becker signalent cependant que les défunts sont parfois classés d'après l'année de leur disparition. Cette disposition fait clairement apparaître les hécatombes de l'été 1914.

41. - DONNAY, Maurice. Centrale. Paris, 1930, p. 105.

42. - BECKER, Annette. Les monuments aux morts. Patrimoine mémoire de la Grande Guerre. Paris, 1988, p. 9.

43. - Voir : BECKER, Annette. Les monuments aux morts. Patrimoine mémoire de la Grande Guerre. Paris, 1988, p. 65-66. L'auteur déplore que l'artiste n'ait pas fait une place aux infirmières sur ce monument. Étant donné sa localisation et la dédicace qu'il porte (Facultas parisiensis suorum memor), cette absence est compréhensible, les infirmières n'étant pas liées à la Faculté de médecine en tant qu'institution (à la différence du personnel administratif).

44. - Arch. nat., 61 AJ 63, École normale supérieure, dossier des commémorations. Discours de Gustave Lanson prononcé lors de l'inauguration du monument aux morts, p. 3.

45. - Arch. nat., 61 AJ 63, École normale supérieure, dossier des commémorations. Discours de Gustave Lanson prononcé lors de l'inauguration du monument aux morts, p. 8.

46. - Arch. nat., 61 AJ 63, École normale supérieure, dossier des commémorations. Discours de Gustave Lanson prononcé lors de l'inauguration du monument aux morts, p. 10.

47. - Arch. nat., 61 AJ 63, École normale supérieure, dossier des commémorations.

48. - Ce texte grec est extrait de Thucydide, Livre II, XLIII : « Des hommes illustres ont pour tombeau la terre entière; ce n'est pas seulement une inscription sur une stèle qui, dans leur pays, rappelle leur existence: même sur le sol étranger, sans rien d'écrit, chacun est habité par un souvenir, qui s'attache à leurs sentiments plutôt qu'à leurs actes " (traduction de Jacqueline de Romilly). Nous remercions monsieur Kaufmann, du centre d'études anciennes de l'École normale supérieure, pour l'identification de ce passage.

49. - Cette salle porte le nom du diplomate qui l'occupa durant sa scolarité à l'école. André François-Poncet exécuta ces peintures en 1908-1909 avec d'autres agrégatifs de l'école (Jean Wahl, Jules Lefèvre-Paul et Charles Avezou) sous la conduite du peintre Pierre-Victor Robiquet. 
Sur cette fresque, voir : CHAPOUTIER, F. « Un monument historique, le problème de l'agrégation de 1909 ». Dans Programme de la Garden-Party. 1956, n. p.

50. - Journal officiel, 21 juillet 1925.

51. - Général Messimy, déclaration au Sénat lors de la discussion d'un article de la nouvelle loi militaire, 31 mars 1928.

52. - Sur cette œuvre disparue, voir: GILLE-DELAFON, S. «Les fresques du réfectoire de l'École centrale des arts et manufactures ». Dans La Construction moderne, 14 janvier 1934, p. 242-247.

53. - GAZIOT, P. «Discours d'inauguration du monument aux morts de l'Institut national agronomique » Dans 1914 -1918, À la mémoire des anciens élèves de l'Institut national agronomique morts pour la défense du sol français. Paris, 1921, p. 20.

54. - «Discours du président de la société de l'École des chartes». Dans L'École des chartes et la guerre, livre d'or orné de cinquante-et-un portraits. Paris, 1921, p. 152-153.

55. - MONFRIN, Jacques. "Discours au nom des élèves de l'école ». Dans Inauguration d'une plaque commémorative des archivistes paléographes et élèves de l'École des chartes victimes de la guerre, 1939-1945. Nogent-le Rotrou, 1946.

56. - GOURDON, H. « Discours du président de l'association des anciens élèves ». Dans Livre d'or de l'École normale supérieure d'enseignement primaire de Saint-Cloud. Paris, 1921, p. 3.

57. - Arch. nat., 61 AJ 63, École normale supérieure, dossier des commémorations. Discours du capitaine Arnaud lors de l'inauguration du monument aux morts de l'École normale.

58. - GOURDON, H. « Discours du président de l'association des anciens élèves ». Dans Livre d'or de l'École normale supérieure d'enseignement primaire de Saint-Cloud. Paris, 1921, p. 3.

59. - 1914-1918, Livre d'or de l'École normale supérieure d'enseignement primaire de Saint-Cloud. Paris, 1921, p. XI.

60. - DONNAY, Maurice. Centrale. Paris, 1930, p. 103.

61. - COSNIER, M. «Discours en la mémoire des agros morts au combat ». Dans Compte-rendu des cérémonies en mémoire des anciens élèves et élèves de l'Institut national agronomique morts pour la France, supplément aux annales de la Société agronomique française et étrangère. Paris, 1917, p. 1.

62. - Je tiens à remercier Sébastien Barret, aujourd'hui chargé de recherche à l'IRHT, pour son aide et ses conseils dans la préparation de ce texte.

63. - Des éléments de cette étude inédite ont été repris dans : « 80 ans de la vie d'un monument aux morts, Le monument aux morts de l'École Normale Supérieure ». Labyrinthe, n5, hiver 2000, 135 p., p. 61-78. Quand la Sorbonne était peinte. Paris : Maisonneuve et Larose, 2001, 304 p.

\section{RÉSUMÉS}

Dans les années suivant la première guerre mondiale, des monuments aux morts ont été érigés dans toutes les communes de France. Paroisses, entreprises, institutions, organismes privés ou publics ont également voulu honorer la mémoire de leurs disparus à travers des monuments "communautaires». Universités et grandes écoles parisiennes ne font pas exception à cette règle. On s'intéresse ici plus particulièrement au mode de financement de ces monuments: conçus en général sans financement public, par le biais de souscriptions, ils révèlent le périmètre de la communauté élargie (familles des disparus, autres écoles, personnages influents, entreprises liées aux écoles). On prendra ensuite en considération les thèmes développés dans les œuvres, en s'attachant à les relier aux autres formes de représentations propres à ces 
établissements, tels que les discours, décors peints ou sculptés... On aura enfin soin de prendre en compte les usages liés à ces œuvres.

INDEX

Mots-clés : grandes écoles, monuments aux morts, première guerre mondiale, universités

\section{AUTEUR}

\section{CHRISTIAN HOTTIN}

Conservateur du patrimoine, adjoint, département du pilotage de la recherche et de la politique scientifique, direction générale des patrimoines, ministère de la Culture et de la Communication christian.hottin@culture.gouv.fr 